\title{
What Is Matter According to Particle Physics, and Why Try to Observe Its Creation in a Lab?
}

\author{
Francesco Vissani 1,2 (D)
}

\section{check for}

updates

Citation: Vissani, F. What Is Matter According to Particle Physics, and Why Try to Observe Its Creation in a Lab? Universe 2021, 7, 61. https:// doi.org/10.3390/universe7030061

Academic Editor: Marek Gazdzicki

Received: 15 February 2021

Accepted: 3 March 2021

Published: 9 March 2021

Publisher's Note: MDPI stays neutral with regard to jurisdictional claims in published maps and institutional affiliations.

Copyright: (C) 2021 by the authors. Licensee MDPI, Basel, Switzerland. This article is an open access article distributed under the terms and conditions of the Creative Commons Attribution (CC BY) license (https:/ / creativecommons.org/licenses/by/ $4.0 /)$.
1 INFN, Laboratori Nazionali del Gran Sasso, 67100 L'Aquila, Italy; vissani@lngs.infn.it; Tel.: +39-346-73-28-009

2 Gran Sasso Science Institute, 67100 L'Aquila, Italy

\begin{abstract}
The standard model of elementary interactions has long qualified as a theory of matter, in which the postulated conservation laws (one baryonic and three leptonic) acquire theoretical meaning. However, recent observations of lepton number violations-neutrino oscillations-demonstrate its incompleteness. We discuss why these considerations suggest the correctness of Ettore Majorana's ideas on the nature of neutrino mass and add further interest to the search for an ultra-rare nuclear process in which two particles of matter (electrons) are created, commonly called neutrinoless double beta decay. The approach of the discussion is mainly historical, and its character is introductory. Some technical considerations, which highlight the usefulness of Majorana's representation of gamma matrices, are presented in the appendix.
\end{abstract}

Keywords: standard model extensions; neutrino masses; Majorana neutrinos; lepton number violation

\section{Introduction}

The discussion of the nature of things is very old. We can experience the wonder of nature, but it is almost inevitable to reason about how to reconcile the evident mutability of what is around us with an equally evident degree of permanence. The hypothesis advanced by Greek atomism is that of a simple and permanent substance, even if not directly perceptible to the senses, which is capable of various arrangements and combinations, that give rise to complex dynamics. It is undeniable that first modern chemistry and then physics have made dramatic contributions to this discussion, to the point of changing the world in which we live.

Here, we shall examine some speculative questions raised by modern particle physics pointing in the same direction. Although they do not have the same practical relevance as those of the "science of the atom" properly said, they still deserve some attention, and not only for speculative reasons. For instance, experimental investigations of these questions have not yet led to clear answers and remain a priority to progress, but they are also becoming significantly more challenging, so it makes sense to assess their interest as accurately as possible. Moreover, as just mentioned, this discussion is part of a glorious tradition.

We will reason about: (1) what particle physics claims about the nature of matter; (2) what conceptual frameworks it gives us to order the available observations; (3) which ones are the most credible, highlighting those that suggest that matter is, to some extent, impermanent. We can already formulate a very precise question to guide the discussion:

$\star$ Is it possible to observe the creation of matter particles in the laboratory?

It is also interesting to keep in mind a somewhat related question: What is the relationship between the hypothetical processes where particles of matter are created and the equally hypothetical processes in which matter is destroyed? In the following discussion, we will focus mostly on the first question. 


\section{Matter and Antimatter in Particle Physics}

\subsection{General Features}

Particle physics inherits directly from atomism the idea that sensible reality can be ideally subdivided into well-defined parts, called (elementary) particles, each with its own characteristics (The Greek word atomos literally means "indivisible"; the Latin word particula means "small part": both concepts refer to atomism. The modern usage has reserved the first word for the basic entities of chemistry and the second for those of fundamental physics.). In the current version, called "relativistic quantum mechanics", particles are described by irreducible representations of finite dimension of the Poincaré group, having certain values of spin and mass, and moreover, each particle is accompanied by an antiparticle (with the same mass and opposite charge). This is true for all particles; let us elaborate on the point of antiparticles, since it is very important for the following.

There are various arguments that show how the combination of relativity and wave mechanics results in the necessity of antiparticles. A very attractive one is due to Feynman [1], while the one we present is closer to Stueckelberg's [2]. It is not particularly elegant, but it has the merit of going straight to the point, clearly highlighting both the undulatory and relativistic bases of the argument.

\subsection{Waves, Relativity, and Charge}

Let us begin from a particle without spin, described by the wave equation of Klein-Gordon:

$$
P^{2} \varphi=m^{2} \varphi \text { where } P_{\mu}=i \hbar \frac{\partial}{\partial x^{\mu}}
$$

In addition to the waves with positive energy $\varphi_{+} \sim e^{-i E t / \hbar}$, there are those with negative energy $\sim e^{+i E t / \hbar}$, which at first sight could seem problematic.

However, they can be usefully interpreted as follows: if we think of them as conjugate waves $\left(\varphi_{-}\right)^{*}$, rather than as a wave of "negative energy" $\varphi_{-}$, as one would instinctively do, we can treat them as outgoing waves-final states of a transition rather than initial states. (A transition dipole evolves in time as $x_{1 \rightarrow 2} \sim e^{i\left(E_{2}-E_{1}\right) t / \hbar}$ (Heisenberg); introducing waves with given energy $\psi \sim e^{-i E t / \hbar}$ (de Broglie), it factorizes in a scalar product between two states $x_{1 \rightarrow 2}=\int d x \psi_{2}^{*} x \psi_{1}$ (Schrödinger). Thus, the interpretation of the conjugate wave as a final state arises at the basic level.) Since the electric charge is included according to the "minimal replacement" principle of Weyl, Fock, etc. (see [3] for a review), i.e.,

$$
P_{\mu} \rightarrow P_{\mu}-q A_{\mu}
$$

and since the electromagnetic four-potential $A_{\mu}$ is real, the wave $\varphi_{-}$obeys the equation with $q \rightarrow-q$, namely it has opposite charge: it is a different particle, with the same mass, but with opposite charge. It is an antiparticle. (We show the factor $\hbar$ explicitly in this section, but will use the system $\hbar=c=1$ elsewhere in these notes).

The very same observation applies to the Dirac equation, that is:

$$
\left(\gamma_{\mu} P^{\mu}-m\right) \psi=0
$$

The argument is simplified by the Majorana representation of gamma matrices [4] (see also Appendix A.1), for which the gamma matrices are purely imaginary, namely:

$$
\gamma_{\mu}^{*}=-\gamma_{\mu}
$$

that we will always use in these notes. In fact, with this choice, we can interpret the "negative energy solutions" just as above:

$$
\psi_{-}^{*} \sim e^{+i E t / \hbar}
$$


From $\left[\gamma_{\mu}\left(P^{\mu}-q A^{\mu}\right)-m\right] \psi_{-}^{*}=0$, we get immediately $\left[\gamma_{\mu}\left(P^{\mu}+q A^{\mu}\right)-m\right] \psi_{-}=0$; or, in other words, there must be solutions with the same mass and opposite charge (=antiparticles). The seemingly simple nature of this argument should not mislead the reader. Even Dirac, who predicted the existence of the anti-electron, needed time to formulate it. (The point of the paper of 1928 [5,6] was to reach a definitive understanding of the spin of the electron. Later, in 1931, he wrote "A hole, if there were one, would be a new kind of particle, unknown to experimental physics, having the same mass and opposite charge to an electron. We may call such a particle an anti-electron". [7]; note in passing that the term "hole", which refers to a specific interpretation, is now largely abandoned.) An appropriately chosen formalism can help; the conceptual point remains nontrivial.

\subsection{Matter Particles}

Let us now focus on those particles that constitute matter. Their prototype is the electron, the first of them to be discovered and presumably the most important in practice, being the one that makes up the shells of atoms. Matter particles:

$\star$ have spin $1 / 2$ and are subject to Fermi-Dirac statistics

$\star$ are divided into two broad classes: quarks [8,9], which have strong interactions and are bound in hadrons; leptons, which do not.

They also satisfy further conservation laws, which we define and discuss next.

In electromagnetic and strong interactions, pairs of particles and antiparticles can be created. This does not imply a violation of the electric charge, and therefore neither a net creation of matter particles. In weak interactions, apparently, the situation is different. It should be stressed that, in the 1930s, Fermi's theory was regarded as revolutionary [10] as it led to thinking about the appearance/disappearance of matter particles for the first time. In fact, the very name beta decay suggests the appearance of an electron in the final state, although this is expressed with terminology from days gone by (that of Rutherford). Let us elaborate on this point, discussing the way in which the naive idea that "each particle be forever" was replaced (This idea was only slowly abandoned. For example, Pauli himself in 1930 believed that neutrinos were contained in the nucleus before decay.) and upgraded by modern particle physics.

After this discovery and even more in the 1940s, with the new world of elementary particles, a number of questions were raised: Why does a proton not disintegrate into an anti-electron and a neutral pion? Why does a muon not transform into an electron and a photon? The fundamental laws of the conservation of energy, angular momentum, and electric charge are compatible with such processes, but they do not happen. The modern formulation of the answer came in 1949 [11], when Wigner proposed to consider the existence of a new law governing the possible transformations of matter, called the conservation of baryonic number: the number of heavy particles, such as protons and neutrons, stays the same in any reaction. A few years later, it was suggested that similar laws apply to leptons [12-14]. (According to T.D. Lee [15], Fermi knew this in advance. Indeed, the same principles had already been anticipated by Weyl [16] and described by Stueckelberg [17], albeit talking of the "number of heavy particles", rather than the "baryon number".)

For instance, returning to weak interactions, let us consider a reference case, neutron decay:

$$
\mathrm{n} \rightarrow \mathrm{p}+\mathrm{e}+\bar{v}_{\mathrm{e}}
$$

The number of baryons is one in the initial and final state, but also, the number of leptons remains unchanged; in fact, the newly created electron counts +1 , but the antineutrino counts -1 , so the net lepton number is zero. We state these laws by saying that the net number of baryons $\mathbf{B}$ never changes (In current understanding, baryons are those bound states that contain three valence quarks. Thus, we can express the same in terms of the net number of quarks; a quark has $\mathbf{B}=1 / 3$.); and in a similar way, the net number of leptons $\mathbf{L}$ is always unchanged. 
$\star$ These laws apply to all known interactions and they are part of the current definition of what matter particles are.

Currently, we know of no exceptions to these rules. Two remarks are in order:

- Experimental tests of $\mathbf{L}$ are less easy than those of $\mathbf{B}$, in particular because of the elusive nature of neutrinos, but they are rather as important, as we will see below.

- There is an interesting theoretical question, about the origin in the known universe of the excess of baryons with respect to the number of anti-baryons.

It is worth noting that both remarks point to major unresolved issues in particle physics; we will come back to that later, showing that $\mathbf{B}$ and $\mathbf{L}$ are much more closely related than it might seem from these empirical considerations.

\section{Are Neutrinos Particles of Matter, or Are They Not?}

\subsection{Majorana's Hypothesis}

Let us consider again Equations (1) and (2). If the particle has no charge, $q=0$, one can identify the waves $\varphi_{+}=\varphi_{-}$; in this case, the particle coincides with its own antiparticle. This is, for instance, what happens in the case of the photon, the particle responsible for electromagnetic interactions. Because of this, the number of such particles may change after a reaction.

What happens to matter (spin 1/2) particles? Let us consider the Dirac equation, Equation (3). In 1937 [4], Majorana remarked that, in principle, if the electric charge of the particle is zero, one could identify:

$$
\psi_{+}=\psi_{-}
$$

using the above notation for the solutions and Majorana's representation of gammamatrices. To put it in simpler words, these special spin 1/2 particles would coincide with their own antiparticles: they would be matter and antimatter at the same time. Majorana suggested that this might be the case for neutrinos or neutrons. Neutrons were discarded because of their magnetic moment [18], but for neutrinos, this hypothesis is still considered plausible and corresponds to very topical questions.

\subsection{The Structure of Weak Interactions}

This observation could confuse a modern reader, accustomed to distinguishing neutrinos from antineutrinos. Obviously, this distinction is not based on the electrical charge of neutrinos, which is not there; so, it is worth going over how we arrived at certain beliefs, to clarify this conceptual point as much as possible.

In 1956, Lee and Yang [19] questioned parity conservation for weak interactions and, shortly after the experiment of $\mathrm{Wu}$ [20], proved the correctness of this supposition. In the next year (1957), independently, Landau, again Lee and Yang, and Salam suggested that neutrinos could have a chiral type of interaction [21-23]. Along with the assumption that their mass is small, this implies that the projection of the spin $\vec{\Sigma}$ in the direction of the momentum $\vec{p}$, i.e., the helicity:

$$
\mathcal{H}=\frac{\vec{\Sigma} \vec{p}}{p}
$$

is negative for neutrinos and positive for antineutrinos. Stating it in plain words: if neutrinos are supposed to be massless, helicity distinguishes them from antineutrinos. This was tested by Goldhaber in 1958 in the lab [24], using ultrarelativistic neutrinos.

Finally, Sudarshan and Marshak [25], and independently Feynman and Gell-Mann [26], generalized the point by suggesting that weak charged currents have a polar-minus-axialvector (i.e., $V-A$ ) structure, which is chiral. The modern reader glimpses one of the main pillars of the standard model behind these positions, but rather than jump too far, it is useful to understand at this point what role neutrino masses play. 


\subsection{Majorana Neutrinos and Weak Interactions}

Now, let us examine what happens if the neutrino mass is not exactly zero. This point was clarified with a bit of difficulty in the scientific literature and has stimulated many interesting results [27-37]. We will discuss this at length later; for now, we would like to limit ourselves to describing the position we have arrived at on the basis of modern physics. We present here a very transparent argument, based on [38]:

Bringing the particles to rest (that is, when the momentum is zero $p=0$ ), the helicity would no longer be defined; we would have only the spin $\vec{\Sigma}$. From the point of view of the spin, the two neutral particles (neutrino and antineutrino) would differ only for the state of rotation; however, an elementary particle should remain unchanged under rotations, which would lead us to think that they are the same particle. (As is well known, we can think of a particle with spin as a sphere without structure, rotating about a vertical axis. The image reflected in a vertical mirror is indistinguishable from the particle with the axis of rotation reversed. The two cases are distinguishable when we have not only spin, but also momentum (and especially in the case where momentum cannot be eliminated, as is the case for a massless particle).) This is consistent with the hypothesis of Majorana.

In principle, we could still invoke some special law to distinguish neutrinos from antineutrinos also in the rest-frame, which would double the number of particles. However, Occam's razor would suggest this is not the first case to consider, as there would be no need for doubling the number of particles. Therefore, we are led to consider seriously the hypothesis of Majorana and to expect that, in the rest-frame, the $V$-Ainteractions would produce the two spin states equally. (The other position, where the number of neutrinos is doubled in the rest system, is the hypothesis that neutrinos are Dirac particles.)

Finally, let us recall the important and well-known observation that neutrino oscillations [30,39,40] cannot distinguish Majorana masses from Dirac masses [31]; we refer to [41] for a further discussion of the hypothesis on the oscillations that interest us in practice, i.e., those occurring in the ultrarelativistic regime.

The above analysis showed that due to the chiral structure of the interactions, when neutrinos are relativistic, the characteristic effects of Majorana neutrinos are suppressed. Further supporting (formal) arguments are presented in Appendix A.2.

In summary, we can say that

- the possibility that the neutrino and antineutrino coincide in the rest-frame-i.e., that Majorana's hypothesis is correct-does not formally contradict what we know;

- most of the empirical knowledge we have about neutrinos concerns only the ultrarelativistic limit instead, and the characteristic manifestations of Majorana hypothesis are suppressed in this limit.

It becomes interesting to understand even better the meaning of this hypothesis (we will discuss it in the next section) and to put it to the test, in the way described in Section 5.

\section{Status of Baryon and Lepton Number Conservation Laws}

We now return to the conservation laws that are part of the definition of what matter is. In recent times, evidence has accumulated that all individual leptonic numbers:

$$
\mathbf{L}_{\mathrm{e}}, \mathbf{L}_{\mu}, \mathbf{L}_{\tau}
$$

are violated, as was discovered through the experiments such as $\mathrm{K} 2 \mathrm{~K}, \mathrm{~T} 2 \mathrm{~K}, \mathrm{NO} v \mathrm{~A}$, and OPERA(see Table 1), which observed the appearance of a lepton of a different type (also known as "flavor", also known as "family"). Instead, as already recalled, there is no empirical evidence that their sum, the total leptonic number,

$$
\mathbf{L}=\mathbf{L}_{\mathrm{e}}+\mathbf{L}_{\mu}+\mathbf{L}_{\tau}
$$

is violated. Majorana's hypothesis for the neutrino evidently violates the leptonic number $\mathbf{L}$ by two units, but as we have discussed, these effects disappear with the neutrino mass. 
We know from observations that neutrino masses are small; the experimental basis for this conclusion is briefly reviewed in the next section just for completeness (as the story is rather well-known), and their implications will be better discussed in the next section. The rest, and the main part of this section, will be devoted to completing the description of the theoretical framework.

Table 1. Selection rules of the observed violations of the exact global symmetries of the standard model.

\begin{tabular}{cccccc}
\hline & $\Delta\left(\mathbf{L}_{\mathbf{e}}-\mathbf{L}_{\mu}\right)$ & $\Delta\left(\mathbf{L}_{\mu}-\mathbf{L}_{\tau}\right)$ & $\Delta\left(\mathbf{L}_{\mathbf{e}}-\mathbf{L}_{\boldsymbol{\tau}}\right)$ & $\Delta(\mathbf{B}-\mathbf{L})$ & Observations \\
\hline$v_{\mu} \rightarrow v_{\mathrm{e}}$ & +2 & -1 & -1 & 0 & {$[42,43]$} \\
\hline$\nu_{\mu} \rightarrow v_{\tau}$ & -1 & +2 & -1 & 0 & {$[44-46]$} \\
\hline
\end{tabular}

\subsection{Neutrino Oscillations and the Evidence of Neutrino Masses}

The only experimental basis for stating that neutrinos have mass is the extensive evidence of neutrino oscillations. The existence of this phenomenon was hypothesized by Bruno Pontecorvo in 1957 [30,47]; at that time, it was called "virtual transition", borrowing the terminology introduced for neutral $K$ mesons [48], and it was (incorrectly) believed that neutrinos transformed into antineutrinos. (For example, we read in [30] the words: IF THE CONSERVATION LAW OF NEUTRINO CHARGE WOULD NOT APPLY, THEN IN PRINCIPLE NEUTRINO $\rightarrow$ ANTINEUTRINO TRANSITIONS COULD TAKE PLACE IN VACUO.) Its description was refined in the following years, leading, 10 years later, to the modern theory years later [49] that exploits the concept of leptonic mixing [50-52]. A further important theoretical ingredient for the interpretation of the data is the "matter effect" [53,54], attributable to an additional phase for $v_{\mathrm{e}}$ and $\bar{v}_{\mathrm{e}}$ due to weak interactions with electrons in ordinary matter.

The first observational substantiation of oscillations was obtained by comparing the solar neutrino data [55-58] with the theory of their production in the Sun [59]. The results of [60] allowed verifying the interpretation in an almost model-independent way. Modern measurements, including those of $[61,62]$, together with KamLAND'sresults with reactor antineutrinos [63] allowed us to progress and measure precisely the relevant parameters, discovering that the electron neutrino is mainly the lightest component of a neutrino mass doublet-a result based on the attestation of the "matter effect".

A second group of observational evidence is given by studies of atmospheric neutrinos. A decisive contribution is attributed to the Kamiokande experiment [64,65], which evolved into the Super-Kamiokande experiment [66]. The results are consistent with many other observations of atmospheric neutrinos, beginning with those of MACRO [67], of SoudanII [68], among others. Again, verifications have been performed under controlled conditions, in particular those obtained thanks to the artificial beams produced in the accelerators [42-44,69] (more on this just below). In addition, further useful information has been obtained thanks to precision measurements in the [70-72] reactors. It is important to investigate the mass spectrum of neutrinos in more detail, to see whether it resembles the mass spectrum of charged fermions or not. At the moment, we only have clues in favor of this option [73-76]; the statistics that will be collected with the next generation of very large detectors [77-79] will provide us conclusive answers. (We will return to the meaning of the mass spectrum later.)

\subsection{Global Symmetries in the Standard Model}

Let us consider the "standard model" of elementary interactions, based on gauge symmetry $\mathrm{SU}(3)_{C} \times \mathrm{SU}(2)_{\mathrm{L}} \times \mathrm{U}(1)_{\mathrm{Y}}$ [80-82] and with the three families of quarks and leptons [83-85]. It is well known that it presents several "accidental symmetries" that imply the conservation laws of $\mathbf{B}, \mathbf{L}_{\mathrm{e}}, \mathbf{L}_{\mu}, \mathbf{L}_{\tau}$, and of course also of their linear combinations such as $\mathbf{L}$, which are valid at the leading (perturbative) level. On the other hand, as just men- 
tioned, the conservation of individual leptonic numbers is not compatible with some experimentally observed facts.

It is even more interesting to observe that the symmetries associated with the specific conservation laws $\mathbf{B}-\mathbf{L}, \mathbf{L}_{\mu}-\mathbf{L}_{\tau}$, and $\mathbf{L}_{\mu}-\mathbf{L}_{\mathbf{e}}$ are exact symmetries instead, i.e., free from quantum anomalies, while $\mathbf{B}$ and $\mathbf{L}$, taken alone, are anomalous [86]. Thus (see again Table 1), various symmetries that would be expected to be exact are violated, and the only (presumably exact) symmetry in the standard model of which we do not know any exception at the moment is:

$$
\mathbf{B}-\mathbf{L}
$$

It is useful to note that a Majorana mass term of neutrinos would mean its violation.

Note in passing that the standard model does predict the existence of baryon number violation manifestations [87] — through non-perturbative phenomena above the electroweak scale, called sphalerons - but they are not sufficient to justify the excess of baryons in the cosmos [88]. This adds interest in physics beyond the standard model related to still unobserved global number violations.

\subsection{Standard Model and Majorana Neutrinos}

At first glance, it would not seem so easy to write a Majorana mass term for neutrinos in the standard model. For example, we can form a Majorana spinor $\chi=v_{\mathrm{L}}+\boldsymbol{v}_{\mathrm{L}}^{*}$ with the ordinary neutrinos $v_{\mathrm{L}}$ and then include in the Lagrangian density also the following Majorana mass term (see the Appendix A for details)

$$
-\frac{m}{2} \chi^{t} \gamma^{0} \chi=-\frac{m}{2} \boldsymbol{v}_{\mathrm{L}}^{t} \gamma^{0} \boldsymbol{v}_{\mathrm{L}}+\text { Hermitian conjugate }
$$

but this term violates a gauge symmetry, the hypercharge $Y$, by one unit. (We use the normalization $Q=T_{3}+Y$, so that $Y=-1 / 2$ for the neutrino field.) On the other hand, this symmetry is broken spontaneously, and with this consideration in mind, we are led to write the following term, which is a perfect gauge invariant:

$$
\boldsymbol{\ell}_{\mathrm{L}}^{t} \varepsilon \boldsymbol{H}=\left(\boldsymbol{v}_{\mathrm{L}}, \boldsymbol{e}_{\mathrm{L}}\right)\left(\begin{array}{cc}
0 & 1 \\
-1 & 0
\end{array}\right)\left(\begin{array}{c}
0 \\
v+\frac{h}{\sqrt{2}}
\end{array}\right)=v \boldsymbol{v}_{\mathrm{L}}+\text { interactions }
$$

where the Higgs doublet $H$ is given in the physical gauge, the expectation value is $v=174 \mathrm{GeV}$, and $\varepsilon=i \sigma_{2}$ is the invariant matrix of $\mathrm{SU}(2)_{\mathrm{L}}$. This term behaves just like the spinorial field under Lorentz transformations, so we can use it to form a term of the Lagrangian density of the type:

$$
\delta \mathcal{L}=-\frac{1}{2 M}\left(\ell_{\mathrm{L} a}^{t} \varepsilon \boldsymbol{H}\right) \gamma_{a b}^{0}\left(\boldsymbol{\ell}_{\mathrm{L} b}^{t} \varepsilon \boldsymbol{H}\right)+\text { Hermitian conjugate }
$$

After spontaneous symmetry breaking, this term reproduces that in Equation (12), therefore yielding Majorana masses. Thus, we identify:

$$
m=\frac{v^{2}}{M} \approx 50 \mathrm{meV} \times \frac{6 \times 10^{14} \mathrm{GeV}}{M}
$$

a relation showing that the neutrino mass values $m$, which have been discovered by means of the neutrino oscillation phenomenon, correspond to very large masses $M$. We note that this mass scale strongly differs from $v=174 \mathrm{GeV}$, the electroweak mass scale, and is smaller than the Planck mass: a valuable indication of new physics.

\subsection{Theoretical Remarks}

As we see, it is allowed to consider Majorana neutrinos in a manner where the gauge symmetries of the standard model are respected, provided that operators of dimension five (i.e., operators that are not renormalizable) are included. Proceeding in this way, the possi- 
ble question of the origin of these masses is postponed to a subsequent, renormalizable formulation of an extension of the standard model, compatible with it.

There is no shortage of attractive theoretical options. The operator in Equation (14) was first considered by Minkowski [89] in a model that includes heavy right-handed neutrinos $v_{R}$, whose quantum fluctuations are suppressed by the inverse of the mass of such particles. Later, it was noted that such a situation is common to several models $[90,91]$ including those with extended gauge symmetries [92,93]. In some of these grand unified theories (GUTs), B - L becomes a (spontaneously broken) gauge symmetry [93-95], while in others, the presence of new particles (including the heavy neutrinos $v_{\mathrm{R}}$ ) offers new possibilities to explain the origin of the baryon asymmetry in the cosmos [96].

For the discussion of observable effects in the lab (which we will conclude in the next section), the most convenient language is that of effective operators, as argued in [97,98]. The operator shown in Equation (14), describing Majorana masses and that violates $\mathbf{L}$ and also $\mathbf{B}-\mathbf{L}$, is unique and is suppressed only by a single power in the mass scale of the new physics. In other words, this is enough to endow the standard model with small Majorana masses of the ordinary neutrinos. Proton decay arises with operators of dimension six; pure baryon number violation phenomena arise with operators of dimension nine, etc. In this scheme, small violations of global numbers are attributed to new physics, which is fully manifested at scales different from those of the standard model. Note that, strictly speaking, none of these effects have been experimentally verified; however, we have indications that neutrinos have mass, and we know that their values are small, which suggests the presence of the dimension five operator just discussed.

Summarizing, we reached the following conclusions:

$\star$ the structure of the standard model does not contradict and in fact revives Majorana's hypothesis: neutrinos, sole among all matter particles, are likely to be their own antiparticles;

$\star$ neutrino masses are expected to be very small, and their value can be regarded as a special observational window on the physics far beyond the standard model itself. (However, it should be noted of the item, as it stands it is horrible. e.g., if the $v_{\mathrm{L}}-v_{\mathrm{R}}$ couplings are small, it is possible to obtain small Majorana masses with lighter $v_{R}$.)

These conclusions are stated in the context of the extended standard model.

\section{Matter Creation in the Lab}

In light of the above considerations, it is more interesting than ever to test the hypothesis that neutrinos have a small Majorana mass, which would make them without equal among matter particles. In fact, neutrinos would be the only elementary particles known to be both matter and antimatter, a hypothesis not only compatible with current theoretical thinking, but even plausible in this context, as just seen above.

Over the years, it has become increasingly clear that the most promising way to validate this hypothesis is to search for a rare nuclear transition in which two neutrons turn into two protons and two electrons, symbolically,

$$
2 \mathrm{n} \rightarrow 2 \mathrm{p}+2 \mathrm{e}^{-}
$$

which, in practice, can be done by studying some nuclear transitions such as ${ }^{76} \mathrm{Ge} \rightarrow{ }^{76} \mathrm{Se}+2 \mathrm{e}^{-},{ }^{130} \mathrm{Te} \rightarrow{ }^{130} \mathrm{Xe}+2 \mathrm{e}^{-},{ }^{136} \mathrm{Xe} \rightarrow{ }^{136} \mathrm{Ba}+2 \mathrm{e}^{-}$, and others; see [99-103] for early works and [104-108] for a few recent reviews. (There is also a useful review of the numerous false starts [109], which illustrate the great desire to be able to measure the aforementioned transition and which above all suggest that one should be cautious before accepting a discovery.) The most important fact for our discussion is that this transition must be thought of, in light of current theories, as: 
$\star$ a violation of the symmetry $\mathbf{B}-\mathbf{L}$ due to the net creation of two matter particles (electrons).

This is the reason why in the rest of this discussion, we can refer to this process as "matter creation". Of course, this is exactly the same process that we use to call "neutrinoless double beta decay" or in similar manners. However, in this discussion, we would like to emphasize its relevance to current (and future) understanding of what matter is according to particle physics, and a new terminology suited for this sake is necessary.

Before outlining the actual connection between Majorana neutrino mass and the process of Equation (16), let us briefly recall how the discussion of this important process has evolved over time, examining the most important conceptual changes.

\subsection{Historical Introduction}

Immediately after Majorana, Racah discussed the interactions of a Majorana particle, showing that weak vector interactions would have physical manifestations distinguishable from those of a Dirac particle [18]. Such a possibility was investigated in 1954, when Davis inquired whether the particle $\bar{v}$ produced in the reactors would trigger ${ }^{37} \mathrm{Cl}+\bar{v} \rightarrow$ ${ }^{37} \mathrm{Ar}+\mathrm{e}^{-}$; the answer turned out to be negative [110]. Later in the literature, the term "Racah chain" was used to refer to a sequence of processes such as this; see [39], which reconstructs the story with great accuracy.

Instead, the specific process of matter creation of Equation (16) was first discussed by Furry in 1939 [111], who was inspired by the process of "double beta disintegration" previously discussed by Goeppert-Mayer (We quote the beginning of the Abstract: THE PROBABILITY OF SIMULTANEOUS EMISSION OF TWO ELECTRONS (AND TWO NEUTRINOS) HAS BEEN CALCULATED. Thus, this is a weak process in which the number of leptons remains unchanged, which using modern notations, can be indicated schematically as $2 \mathrm{n} \rightarrow 2 \mathrm{p}+2 \mathrm{e}^{-}+2 \bar{v}_{\mathrm{e}}$ (compare with Equation (16)); today, this is often denoted "double beta with two-neutrinos", and it has been measured for various nuclei.) [112] and of course by Majorana's ideas. However, it is important to repeat that the theory of weak interactions differed in a crucial manner from the one we have today. If Fermi's vector interaction had been correct in the strict sense, as originally thought, the Majorana hypothesis would have had a much greater effect on the process of the creation of matter. (This would also have been true for any other interaction except for a chiral theory). This was Furry's expectation. (The technical discussion in Appendix A.2 and Appendix A.3 applies to the current theory of weak interactions instead.)

Table 2 illustrates the fortunes of the works $[4,18,27,111,112]$ over time; the last paper is included for reasons explained in Appendix A.4. Even if it is indisputable that the number of scientific publications is growing faster and faster, it seems difficult to deny the fact that the greatest interest in Majorana's work arose in relatively recent times. In order to understand better the meaning of this late outburst of interest, let us begin by identifying three main periods:

- the period of theoretical novelty, in which the hope of observing very large effects was highlighted by Furry and Majorana's neutrinos were actively discussed by scientists such as Stueckelberg, Kemmer, Touschek, Pauli, Harish-Chandra, Michel, Yang, Tiomno, etc.;

- the period begun by the understanding of the $(V-A)$ structure of weak interactions, where, on the other hand, there was an exaggeration in the opposite direction, and doubt was cast whether Majorana's theory should be dismissed-more in Appendix A.4;

- the current period, where the stress shifted from the concept of Majorana's neutrinos to the more precise one of Majorana neutrino masses, subsequently including their meaning in the context of the extensions of the standard model.

(Compare with the much more detailed tables in Pontecorvo's review work [39], already cited, and with the valuable historical study [113]. The Introduction to the book [114] also has a table comparable with Table 2; the main difference is that it lacks a discussion of 
the second, crucial period, which we present in this section and complete in Appendix A.4. (On the other hand, the book includes a collection, albeit incomplete, of many relevant articles useful for the discussion in which we are interested.))

Table 2. The number of papers per decade citing a few seminal articles, including Majorana's, from their appearance to the present day. In the last two lines, a (incomplete) list of theoretical and observational facts that are of major relevance for current discussion. From https://inspirehep.net/ (accessed on December 2020). GUT, grand unified theory.

\begin{tabular}{cccccccccc}
\hline & '30s & '40s & '50s & '60s & '70s & '80s & '90s & $\mathbf{2 0 0 0}$ & $\mathbf{2 0 1 0}$ \\
\hline Majorana [4] & 3 & 3 & 8 & 5 & 17 & 43 & 67 & 159 & 745 \\
\hline Goeppert-M. [112] & 2 & 2 & 6 & 0 & 0 & 18 & 19 & 41 & 221 \\
\hline Racah [18] & 2 & 1 & 6 & 1 & 6 & 19 & 16 & 31 & 133 \\
\hline Furry [111] & 0 & 2 & 6 & 0 & 1 & 25 & 30 & 71 & 351 \\
\hline Case [27] & - & - & 1 & 10 & 10 & 36 & 43 & 34 & 36 \\
\hline theory & $v$ & $\mathbf{B}, \mathbf{L}$ & $V-A, \mathrm{SU}(2)$ & SM, oscill. & $\mathrm{SM}$ & GUT, $v_{\odot}$ & SUSY & glob.anal. & cosm. \\
\hline exp. and obs. & $\mathrm{n}, \mathrm{e}^{+}, \mu$ & $\pi, K$ & $v, V-A$ & $v_{\odot}$ anom. & $\mathrm{SM}, v_{\odot}$ & $W, Z^{0}, v_{\mathrm{atm}}$ & oscill. & oscill. & Higgs, cosm. \\
\hline
\end{tabular}

Let us add a few comments to put this historical examination in the perspective of the discussion in which we are interested. Rather plausible theoretical ideas lead us to think that neutrinos have small masses of the type considered by Majorana. Furthermore, the existence of neutrino oscillation phenomena, predicted in $[49,53,54]$, is currently widely recognized, also by the 2015 Nobel prize in physics, and it has been verified in controlled experiments, in particular those mentioned in Table 1 . This leads to a firm conclusion that neutrino masses are not zero. (From this point of view, it should be stressed that an activity whose significance in theoretical physics might seem apparently modest, that of the global analysis of neutrino oscillation data, has acquired instead an enormous importance since the 1990s, and this is the reason why we mention it explicitly in the table above: this is the only way to date to measure the neutrino mass parameters. See, e.g., $[115,116]$, which have witnessed a continued effort over a period of about 25 years.) Thus, a combination of theoretical and experimental considerations led us to think, today, that the rate of the decay process first considered by Furry (that we regard as a matter creation process) is controlled by the value of Majorana's neutrino mass.

These considerations justify the enduring efforts in testing this process in the laboratory. In fact, several important experiments of this kind have already been carried out, and new efforts are underway; see [117-120] for a few recent experimental searches and $[38,106-108]$ for reviews.

\subsection{What We Know about the Relevant Majorana Neutrino Mass}

The structure of the standard model is compatible with the idea of very small Majorana neutrino masses, to be attributed to new physics, at much larger energy scales. This suggests that there are other physical manifestations, but that they are not (easily) detectable at low energies, and conversely, that makes it even more important to carefully plan those measurements that can be actually performed, based on the hypothesis that the leading reason for the electron/double beta creation rate of Equation (16) is the Majorana neutrino masses. A correct evaluation of nuclear matrix elements is essential to obtain a prediction of the rate, and as is well known, this implies uncertainties, not yet fully clarified. However, the main reason for the uncertainty in the prediction remains simply that due to the value of the Majorana mass that is relevant for this process. Therefore, we will conclude by addressing the quantitative aspects, which are brought into play by these theoretical assumptions.

Suppose that the three ordinary neutrinos have the Majorana mass. We will have the following Lagrangian density (The complex numbers $m_{\ell \ell^{\prime}}$ form a symmetric matrix; the proof is in Equation (A8). Its decomposition can be presented as a relation among 
matrices: $\left.\hat{m}=\hat{U}^{*} \operatorname{diag}\left(m_{1}, m_{2}, m_{3}\right) \hat{U}^{\dagger}.\right)$ :

$$
\delta \mathcal{L}=-\frac{m_{\ell \ell^{\prime}}}{2} \boldsymbol{v}_{\mathrm{L} \ell}^{t} \gamma^{0} \boldsymbol{v}_{\mathrm{L} \ell^{\prime}}+\text { h.c., where } m_{\ell \ell^{\prime}}=U_{\ell i}^{*} m_{i} U_{\ell^{\prime} i}^{*}
$$

where the indices are $\ell, \ell^{\prime}=\mathrm{e}, \mu, \tau$ and point to its components in the "flavor space"; $m_{i} \geq 0$ are the neutrino masses, and $U_{\ell i}$ is the leptonic mixing matrix. As is evident from the selection rule on the electronic lepton number $L_{e}$, the quantity that matters for the transition of Equation (16) is only the matrix element $m_{\mathrm{ee}} \equiv m_{\beta \beta}$, which can be chosen to be real and positive; see again Appendix A.3.

The neutrino oscillation experiments (see Section 4.1) have opened measuring very precisely the absolute values of $U_{\ell i}$ and the absolute value of the differences $m_{i}^{2}-m_{j}^{2}$. The sign of the latter quantity is known precisely for the two masses closest to each other (called $m_{1}$ and $m_{2}$ ), while we do not know for sure whether $m_{3}$ is larger or smaller than the other two masses. To be well defined, we will focus on the case $m_{1}<m_{2}<m_{3}$. The experimental evidence, albeit weak, is currently in favor of this position. In this way, the mass spectrum of neutrinos more closely resembles that of charged fermions and is therefore called the "normal mass spectrum" (also known as "mass hierarchy" also known as "mass ordering").

The real trouble is that:

the oscillations probe neither the phases of $U_{\ell i}$ nor the mass of the lightest neutrino $m_{1}$.

This implies that the parameter $m_{\mathrm{ee}}$ can range from zero to the maximum value allowed by experimental measurements. The theory of fermion masses (and in particular, the one of neutrino masses) is not sufficiently developed to be reliable, and it is difficult to obtain further useful information.

To proceed on an empirical basis, the following steps were taken. In order to address the problem of the phases (sometimes called "Majorana phases"), it was proposed in [121] to consider the maximum and minimum value of $m_{\mathrm{ee}}$, by varying the unknown complex phases, obtaining the formulas:

$$
\begin{aligned}
& m_{\mathrm{ee}}^{\mathrm{max}}=\sum_{i=1}^{3}\left|U_{\mathrm{e} i}^{2}\right| m_{i} \\
& m_{\mathrm{ee}}^{\min }=\max \left\{2\left|U_{\mathrm{e} i}^{2}\right| m_{i}-m_{\mathrm{ee}}^{\max }, 0\right\}
\end{aligned}
$$

See Figure 1 for the standard presentation. (The average value $\left(m_{\mathrm{ee}}^{\max }+m_{\mathrm{ee}}^{\min }\right) / 2$ shown in Figure 1 has a simple analytical expression; e.g., for large $m_{1}$, it is just $\left|U_{\mathrm{e} 1}^{2}\right| m_{1}$, in which the mixing parameter $U_{\mathrm{e} 1}$ is almost perfectly known and $m_{1}$ is not at all.)

The second problem, namely the fact that the mass of the lightest neutrino is unknown, can be tackled in two ways:

- In principle, by means of extremely precise measurements of the absolute neutrino mass $m_{v_{\mathrm{e}}}^{2}=\sum_{i}\left|U_{\mathrm{e} i}^{2}\right| m_{i}^{2}$ (a parameter discussed in [122-126]) in the laboratory. Current experiments indicate $m_{v_{\mathrm{e}}}<0.8-1.1 \mathrm{eV}$ at $90 \%$, and the sensitivity will reach $200 \mathrm{meV}$ in the near future [127]. The current limit directly translate into $m_{1}<0.8-1.1 \mathrm{eV}$.

- $\quad$ From cosmology measurements that probe $\Sigma=m_{1}+m_{2}+m_{3}$, as suggested in [128]. Even if they are based on a cosmological model that is conceptually complex and still under verification, the sensitivity of the most recent measurements is just as impressive: see, e.g., [129-131]. Proceeding as in [132,133], we get $\Sigma<100$ [150] meV at 1 [2] $\sigma$, which translates into $m_{1}<20$ [40] $\mathrm{meV}$.

An empirical approach based on cosmological measurement results in an upper bound of $m_{\mathrm{ee}}$ of a few $10 \mathrm{meV}$. This result, obtained and discussed in [132-135], reinforces the view stated in [38] that it will be a real challenge to be able to observe the electron creation process of Equation (16). In particular, we can already conclude on this basis that detectors with a mass of many tonnes, and able to operate under stable conditions for many years, 
will be needed in order to have the chance of detecting the transition due to the Majorana mass of ordinary neutrinos, as argued in [38].
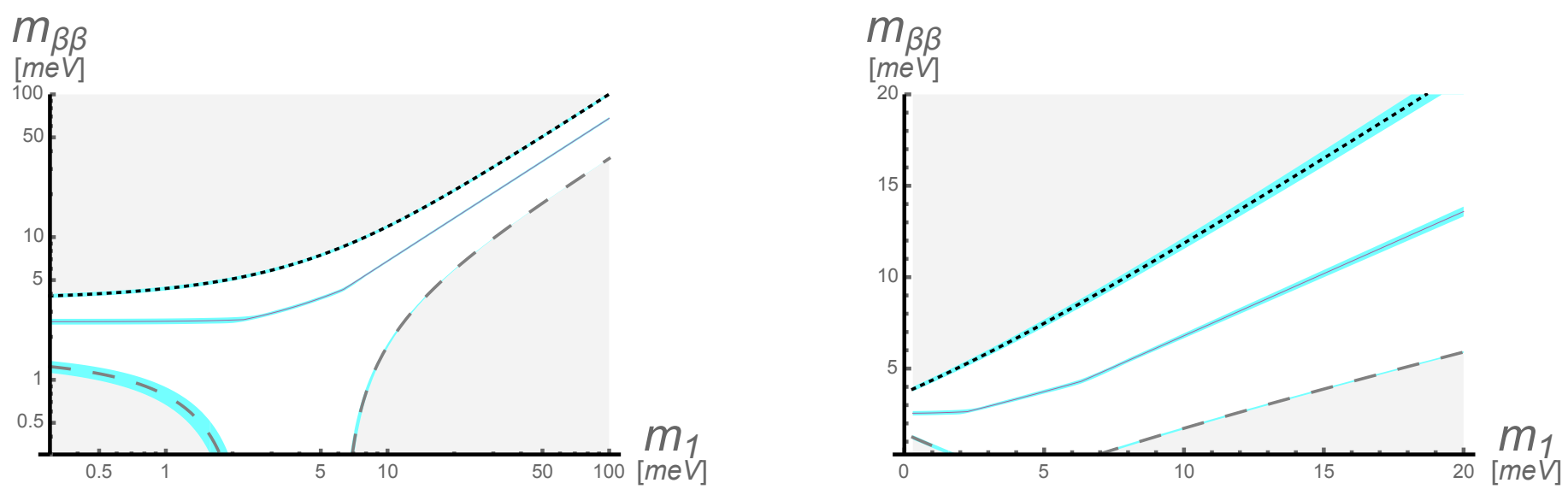

Figure 1. Allowed region for the parameter $m_{\beta \beta}=m_{\mathrm{ee}}$ (white) assuming a normal mass spectrum. On the left, log-log graph; on the right, zoom in the region of the smallest $m_{1}$ somewhat favored by cosmology (see the text and Figure 4 of [134]). The central curve is the average value. The colored bands indicate the uncertainty regions (one sigma). Gray regions are incompatible with observed neutrino oscillations.

\section{Summary and Discussion}

In this paper, we retrace some important steps in the understanding of what matter is, according to modern particle physics. We show how Majorana's ideas are rooted right at the heart of the discussion about the question of whether it is possible to create particles of matter. Trying to summarize, we could say that the search for the value of Majorana's neutrino mass is gaining momentum. (A side comment concerns some basic techniques proposed in [4] and illustrated in the Appendix A, which still retain a certain practical usefulness.)

We reiterate that we have taken care to touch on the main historical lines, but it is important to be aware that at any given time, there are forward-looking ideas, rearguard discussions, and even missteps. Obviously, it is not easy to correctly identify one's position in the present, i.e., at the moment when history is being made, but we believe that having a correct perception of the road already traveled can help keep moving in the right direction. In this spirit, and by way of summary, we provide in Table 3 a schematic description of the evolution of the ideas about what matter is.

We stress that this review has no claim to completeness and that we have left aside many interesting and widely discussed topics in the scientific literature. Just as a specific example, let us consider the idea that the transition proposed by Furry, Equation (16), is not due to the Majorana masses of neutrinos, but rather to some other cause. This hypothesis has also been examined and thoroughly discussed: for the first time by Touschek in 1948 [99] (see also [113,136]), then just after the discovery of the chiral structure of weak interactions [137], again after the understanding of neutrino oscillations [138], and many other times recently. It is not possible to rebut it on an empirical basis, and current theories are not in a position to exclude it absolutely. Nevertheless, it does not seem possible to argue that it has the same interest as Majorana's hypothesis, especially in the context of motivated extensions of the standard model, and unless the scale of new physics turns out to be unexpectedly low.

To conclude this discussion, we would like to express an impression somewhat conveyed by this brief historical review: while it is certainly important (essential) to have new experimental facts to discuss, it seems at least as important to have clear and well-defined theoretical ideas to enable real progress, and it is essential to be able to acknowledge and value them (perhaps not a very strong point, but one on which it is useful to work). 
Finally and quite importantly, it must be said that there is no shortage of tasks for the future, and most of them are quite demanding. In fact, efforts will be needed, devoted: (1) to consolidate inferences about the mass of the neutrino (by means of cosmology and laboratory investigations); (2) to continue and to refine the experimental search for the nuclear transition in which two particles of matter (= electrons) are created; (3) to make progress in the evaluation of the nuclear matrix elements related to this transition, quantitatively estimating the errors in expectations; (4) and last but not least, to develop convincing and principled extensions of the standard model, which allow us to obtain useful and reliable indications on the expected value of the Majorana neutrino mass.

Table 3. Table of elementary components of matter according to different models, successive in time. Left columns: brief description. Right ones: shortcomings of the given model. Dates and information [in brackets] refer to mainly theoretical considerations. The current model of what matter is is the one in the penultimate line, since the Majorana mass hypothesis is very reasonable, but does not yet enjoy observational evidence; to date, the limitations of the last model are only speculative.

\begin{tabular}{|c|c|c|c|}
\hline $\begin{array}{r}\text { Matter } \\
\text { Components }\end{array}$ & $\begin{array}{r}\text { Salient } \\
\text { Features }\end{array}$ & $\begin{array}{l}\text { Valid } \\
\text { Until }\end{array}$ & $\begin{array}{l}\text { Reason for } \\
\text { Inadequacy }\end{array}$ \\
\hline atoms & species, mass & [1838] 1909 & [atoms of electricity] electron \\
\hline nuclei and $\mathrm{e}^{-}$ & charge, mass, spin & [1930] 1956 & [Fermi th.] neutrons and neutrinos \\
\hline $\mathrm{p}, \mathrm{n}, \mathrm{e}, v_{\mathrm{e}}, \mu, \ldots$ & $\mathbf{B}, \mathbf{L}_{\mathrm{e}}, \ldots, ", "$ & [1961] 1968 & [standard model] quarks \\
\hline quarks and leptons & B-L, $\mathbf{L}_{\mathrm{e}}-\mathbf{L}_{\mu}, \mathbf{L}^{-}-\mathbf{L}_{\tau}, ",{ }^{\prime}, "$ & [1962] 2010 & [lepton mixing] appearance exp. \\
\hline quark/antilepton & B-L, ", ," & [1937] ? & [Majorana mass] $2 \mathrm{n} \rightarrow 2 \mathrm{p}+2 \mathrm{e}$ \\
\hline fermions & mass, spin & [1977?] ??? & [supersymmetry?] ??? \\
\hline
\end{tabular}

Funding: This work is partially supported by Research Grant Number 2017W4HA7S “NAT-NET: Neutrino and Astroparticle Theory Network" under the program PRIN 2017 funded by the Italian Ministero dell'Istruzione, dell'Università e della Ricerca (MIUR).

Acknowledgments: I am grateful to Clementina Agodi, Giovanni Benato, Corrado Caselunghe, Silvia de Bianchi, Stefano Dell'Oro, and Adriano Di Giovanni for useful discussions. This work is partly based on the presentations [139-141].

Conflicts of Interest: The author declares no conflict of interest.

\section{Appendix A. On Majorana Spinors}

In this Appendix, we cover several technical aspects concerning Majorana spinors [4]. Here (as in the rest of this paper), we consistently use Majorana's representation of the gamma-matrices just as advocated, e.g., in [34]. For further discussions, see [38,41]. After the introduction, Appendix A.1 and Appendix A.2, we present in Appendix A.3 the definition of the key parameter, $m_{\mathrm{ee}}$. A major obstruction for a useful discussion of Majorana's neutrino mass, which persisted for more than twenty years, is examined critically in Appendix A.4.

\section{Appendix A.1. Illustration of the Usefulness of Majorana Representation}

Thanks to the characteristic feature of the Majorana representation of gamma-matrices, all relevant $4 \times 4$ matrices have simple properties under conjugation and transposition, given in Table A1. This is rather useful in various manipulations.

First example:

Let us begin by showing the consistency of Majorana's condition for a spinor,

$$
\chi=\chi^{*}
$$


The generic Lorentz transformation is parameterized by three angles $\theta_{i}$ and three rapidities $\eta_{i}$ (This parameter is connected to the velocity by $\beta=\tanh \eta$, so that $t^{\prime}=$ $\gamma(t+\beta x)=\cosh \eta t+\sinh \eta x)$ :

$$
\begin{gathered}
\delta \chi=\frac{i}{2}(\vec{\Sigma} \vec{\theta}+\vec{K} \vec{\eta}) \chi \\
\delta \chi^{*}=\frac{i}{2}\left(-\vec{\Sigma}^{*} \vec{\theta}-\vec{K}^{*} \vec{\eta}\right) \chi^{*}
\end{gathered}
$$

Since the spin and the boost matrices are both imaginary, as shown in Table A1, it is evident that $\chi$ and $\chi^{*}$ transform in the same way.

Table A1. Properties of gamma-matrices in Majorana representation. We define as usual $\gamma^{0}=\beta$ and $\vec{\gamma}=\beta \vec{\alpha}$ from Dirac's Hamiltonian; $\gamma^{5}=i \gamma^{0} \gamma^{1} \gamma^{2} \gamma^{3}$ as the chirality matrix; $\Sigma_{j k}=i \gamma_{j} \gamma_{k}$. Generators of Lorentz's group: $\Sigma_{1}=\Sigma_{23}, \Sigma_{2}=\Sigma_{31}, \Sigma_{3}=\Sigma_{12}$ (spin), and $\vec{K}=i \gamma^{0} \vec{\gamma}=i \vec{\alpha}$ (boost). The indices $i, j, k$ take the values $1,2,3$.

\begin{tabular}{cccccccc}
\hline & $\gamma^{0}$ & $\vec{\gamma}$ & $\gamma^{5}$ & $\beta$ & $\vec{\alpha}$ & $\vec{\Sigma}$ & $\overrightarrow{\boldsymbol{K}}$ \\
\hline Hermiticity & + & - & + & + & + & + & - \\
reality & - & - & - & - & + & - & - \\
symmetry & - & + & - & - & + & - & + \\
\hline
\end{tabular}

\section{Second example:}

As a second example of application, let us consider the familiar Lagrangian density of a Dirac field $\psi$ (recall that $\hbar=c=1$ ):

$$
\mathcal{L}=\bar{\psi}\left(i \gamma^{\mu} \partial_{\mu}-m\right) \psi=\psi^{\dagger}\left(i \partial_{0}-i \vec{\alpha} \vec{\nabla}-m \beta\right) \psi
$$

where $\mu=0,1,2,3, \partial_{\mu}=\partial / \partial x^{\mu}$ and $(\vec{\nabla})^{i}=\partial^{i}$ with $i=1,2,3$. We would like to rewrite $\mathcal{L}$ with Majorana's fields, still using Majorana's representation of gamma-matrices. Thus, let us express the Dirac field as follows,

$$
\psi_{a}=\frac{\chi_{a}+i \lambda_{a}}{\sqrt{2}} \text { where } \chi^{*}=\chi, \lambda^{*}=\lambda
$$

which resembles closely the separation of a complex number into its real and imaginary parts. Various mixed terms appear, but we note that, owing to the anticommutativity of the fermionic fields, they are either zero or "surface terms" that can be omitted in the action $S=\int d^{4} x \mathcal{L}$

$$
\begin{gathered}
\chi^{t} \partial_{0} \lambda-\lambda^{t} \partial_{0} \chi=\partial_{0}\left(\chi^{t} \lambda\right) \\
\chi^{t} \alpha^{i} \partial_{i} \lambda-\lambda^{t} \alpha^{i} \partial_{i} \chi=\partial_{i}\left(\chi^{t} \alpha^{i} \lambda\right) \\
\chi^{t} \beta \lambda-\lambda^{t} \beta \chi=\chi^{t} \beta \lambda-\chi^{t} \beta \lambda=0
\end{gathered}
$$

Therefore, the result is equivalent to two decoupled Lagrangian densities,

$$
\mathcal{L}=\frac{1}{2} \bar{\chi}\left(i \gamma^{\mu} \partial_{\mu}-m\right) \chi+\frac{1}{2} \bar{\lambda}\left(i \gamma^{\mu} \partial_{\mu}-m\right) \lambda
$$

Third example:

From the last formula, we read the Majorana mass term:

$$
-\frac{m}{2} \bar{\chi} \chi=-\frac{m}{2} \chi^{t} \gamma^{0} \chi
$$

a simple expression that applies for Majorana's form of gamma matrices. Now, we verify explicitly that this term is non-zero, using the components. Let us consider a term in a Lagrangian density with two spinors:

$$
\chi_{a} \gamma_{a b}^{0} \lambda_{b}=-\lambda_{b} \gamma_{a b}^{0} \chi_{a}=\lambda_{b}\left(-\gamma^{0}\right)_{b a}^{t} \chi_{a}=\lambda_{b} \gamma_{b a}^{0} \chi_{a}
$$


where in the first step we used the anticommutativity of the spinors, which are quantized fermionic fields, and in the last passage, we use the antisymmetry of $\gamma^{0}$, pointed out in Table A1. This calculation might seem pedantic; however, it is not entirely useless. For example, consider one statement of [142]: “THE SCALAR $\bar{\psi} \psi$ OF THE MAJORANA THEORY VANISHES IDENTICALlY"; when we replace $\chi=\lambda=\psi$ in Equation (A8), we see that this statement has no valid basis, and we can rebut it.

Finally, treating the fields as matrices and applying the usual rules for Hermitian conjugation, namely $(\boldsymbol{a} \boldsymbol{b})^{*}=\boldsymbol{b}^{*} \boldsymbol{a}^{*}$, we can show that the same term is Hermitian (real),

$$
\left(\chi_{a} \gamma_{a b}^{0} \lambda_{b}\right)^{*}=\lambda_{b}^{*}\left(\gamma_{a b}^{0}\right)^{*} \chi_{a}^{*}=\lambda_{b}\left(\gamma_{a b}^{0}\right)^{*} \chi_{a}=-\lambda_{b} \gamma_{a b}^{0} \chi_{a}
$$

In the second passage, we used the reality condition of Majorana spinors and in the third the fact that $\gamma^{0}$ is imaginary. Then, applying the same manipulations as in Equation (A8), we conclude the proof of Hermiticity-which implies the unitarity of the time evolutor.

Fourth example:

Let us consider the coupling of a fermion of Majorana with an external electromagnetic field. In the case of an electrostatic interaction, the Hamiltonian density that describes the interaction is:

$$
\mathcal{H}=q A_{\mathrm{ext}}^{\mu} \bar{\psi} \gamma_{\mu} \psi=q \varphi \rho_{\mathrm{ext}} \text { where } \rho_{\mathrm{ext}}=\psi^{\dagger} \psi
$$

Owing to Majorana's condition, Equation (A1), $\psi^{\dagger} \psi=\psi^{t} \psi=\psi_{a} \psi_{a}=0$ due to anticommutativity. Next let us consider the case of magnetic coupling, namely the Hamiltonian density:

$$
\mathcal{H}=\frac{m}{4} F_{\text {ext }}^{\mu \nu} \bar{\psi} \Sigma_{\mu \nu} \boldsymbol{\psi}=-m \vec{B}_{\text {ext }} \vec{S} \text { where } \vec{S}=\frac{1}{2} \psi^{\dagger} \gamma^{0} \vec{\Sigma} \boldsymbol{\psi}
$$

Now, using Table A1, it is easy to prove that the following combination of matrices is symmetric $\left(\gamma^{0} \vec{\Sigma}\right)^{t}=\vec{\Sigma}^{t}\left(\gamma^{0}\right)^{t}=\vec{\Sigma}^{0}=\gamma^{0} \vec{\Sigma}$; thus, the spin operator $\vec{S}$ is zero for a Majorana field, again due to the anticommutativity of the fermionic fields.

\section{Appendix A.2. Dirac and Majorana Mass in One-Neutrino Transitions}

We discuss that weak charged interactions, due to their chiral $(V-A)$ character, and distinguish neutrinos and antineutrinos in the limit where their masses are zero, as in the standard model, and also in the case in the ultrarelativistic limit, if their masses are not zero, as is the case. This circumstance in practice limits the possibilities of distinguishing Dirac's neutrinos from Majorana's, as we discuss below.

Let us begin by considering the formalism of quantum fields in the case of a Dirac neutrino:

$$
\boldsymbol{v}_{\text {Dirac }}(t, \vec{x})=\sum_{\vec{p} \lambda}\left[\boldsymbol{a}_{\vec{p} \lambda} \psi_{\vec{p} \lambda}(t, \vec{x})+\boldsymbol{b}_{\vec{p} \lambda}^{\dagger} \psi_{\vec{p} \lambda}^{*}(t, \vec{x})\right]
$$

where $\lambda= \pm 1$ is the helicity of the states and $\psi$ are bispinors of plane waves with positive energies: $\psi_{\vec{p} \lambda}(t, \vec{x})=u_{\vec{p} \lambda} e^{-i(E t-\vec{p} \vec{x}) / \hbar}$, the first term describing the creation of a neutrino in the final state and the second term describing the disappearance of an antineutrino in the initial state. We used Majorana's representation of $\gamma$ matrices, and thus, the chirality matrix $\gamma^{5}=i \gamma^{0} \gamma^{1} \gamma^{2} \gamma^{3}$ is also imaginary. When we consider a weakly charged interaction, the quantized field is multiplied by the projector $P_{L}=\left(1-\gamma^{5}\right) / 2$. As is well known (see, e.g., $[38,41])$ for ultrarelativistic neutrinos, the chiral projector selects only bispinors with negative helicity, $P_{L} \psi_{\vec{p} \lambda} \approx \psi_{\vec{p} \lambda}$ if $\lambda=-1$ and $P_{L} \psi_{\vec{p} \lambda} \approx 0$ if $\lambda=+1$. In correspondence, we have the matrix elements:

$$
\left\langle v_{\vec{p} \lambda}\left|P_{L} v_{\text {Dirac }}(t, \vec{x})\right| 0\right\rangle \approx\left\{\begin{array}{ll}
\psi_{\vec{p}} \lambda & \text { if } \lambda=-1 \\
0 & \text { if } \lambda=+1
\end{array} \text { when }|\vec{p}| \gg m c\right.
$$


For antineutrino states, we have $P_{L} \psi_{\vec{p} \lambda}^{*}=\left(P_{R} \psi_{\vec{p} \lambda}\right)^{*}$, and so, antineutrino states with positive (opposite to neutrino) helicity are selected. Neutrinos with positive helicity and antineutrinos with negative helicity do not interact at all; they are sterile.

For Majorana fields, the only change is to identify the operators as follows (This can also be formally achieved by imposing $\boldsymbol{v}_{\text {Majorana }}=\left(\boldsymbol{v}_{\text {Dirac }}+\boldsymbol{v}_{\text {Dirac }}^{*}\right) / \sqrt{2}$, thus $c=(\boldsymbol{a}+\boldsymbol{b}) / \sqrt{2}$.):

$$
v_{\text {Majorana }}(t, \vec{x})=\sum_{\vec{p} \lambda}\left[c_{\vec{p} \lambda} \psi_{\vec{p} \lambda}(t, \vec{x})+c_{\vec{p} \lambda}^{\dagger} \psi_{\vec{p} \lambda}^{*}(t, \vec{x})\right]
$$

The argument exposed in Equation (A13) for the Dirac field continues to apply, with two crucial differences

- at the order $m / p$, we can have admixtures with the "wrong type" of particles (antineutrinos rather than neutrinos or vice versa);

- the operators corresponding to the "sterile" states are completely absent.

Therefore, due to the structure of the weakly charged interactions, in the usual case of ultra-relativistic neutrinos (when the mass of neutrinos is small compared to their momentum), the differences between the two types of quantized fields disappear.

\section{Appendix A.3. Electron Creation and Parameter $m_{\mathrm{ee}}$}

Consider the semi-leptonic Hamiltonian density leading to the emission of an electron $\mathcal{H}=\sqrt{2} G_{\mathrm{F}} \boldsymbol{J}_{\mu}^{+} \boldsymbol{j}^{-\mu}$, where the leptonic current is:

$$
j_{\mu}=\overline{\boldsymbol{e}} \gamma_{\mu} \boldsymbol{v}_{\mathrm{Le}}=\sum_{j=1}^{3} U_{\mathrm{e} j} \overline{\boldsymbol{e}} \gamma_{\mu} P_{\mathrm{L}} \chi_{j} \text { with } \chi_{j}=\chi_{j}^{*}
$$

where we postulated that the neutrino mass eigenstates are Majorana fields. The leptonic part of the amplitude, which describes the creation of a couple of electrons, is $\left\langle\mathrm{e} \mathrm{e}\left|T\left[j_{v}(x) \boldsymbol{j}_{\mu}(y)\right]\right| 0\right\rangle$, and it requires evaluating the contraction $\left\langle 0\left|T\left[\boldsymbol{v}_{\mathrm{Le}}(x) \boldsymbol{v}_{\mathrm{Le}}^{t}(y)\right]\right| 0\right\rangle$, namely an unusual type of propagator, which however is non-zero in Majorana's theory. In fact, from $v_{\mathrm{Le}}=U_{\mathrm{ej}} P_{\mathrm{L}} \chi_{j}$, used above, and its transpose, written as $v_{\mathrm{Le}}^{t}=U_{\mathrm{e} j} \bar{\chi}_{j} P_{\mathrm{L}} \gamma^{0}$, the core of the problem reduces to the calculation of an ordinary propagator, namely $\left\langle 0\left|T\left[\chi_{j}(x) \bar{\chi}_{j}(y)\right]\right| 0\right\rangle$. The result is:

$$
\left\langle 0\left|T\left[v_{\mathrm{Le}}(x) \boldsymbol{v}_{\mathrm{Le}}^{t}(y)\right]\right| 0\right\rangle=P_{\mathrm{L}} \gamma^{0} \int \frac{d^{4} p}{(2 \pi)^{4}} \frac{i U_{\mathrm{e} j}^{2} m_{j} e^{-i p(x-y)}}{p^{2}-m_{j}^{2}+i 0^{+}}
$$

The virtual momentum in the denominator has a small time component due to kinematical constraints, whereas the spatial component is of the order of the radius $|\vec{p}| \sim 1 / R_{0}$; therefore, the masses of the light neutrinos $m_{j} \ll 100 \mathrm{meV}$ are absolutely negligible in the denominator, and the lifetime will depend on neutrino masses and mixing only through:

$$
m_{\mathrm{ee}}=\left|\sum_{j=1}^{3} U_{\mathrm{ej}}^{2} m_{j}\right|=m_{\beta \beta}
$$

We use the same symbols as the ee-element of the neutrino mass matrix, since the two quantities coincide with the phase choice that makes the ee-element real and non-negative. The same quantity is sometimes called "effective neutrino mass" or also "electron neutrino mass", most often indicated with the symbol $m_{\beta \beta}$, which recalls the term "(neutrinoless) double beta decay", but which does not emphasize the connection with neutrino masses. In this way, we covered the key topic, which started ideally with [27] in modern times (after $V-A$ ) and which was fully completed in [33], with the introduction of $m_{\mathrm{ee}}$.

To summarize, it is only possible to distinguish between Dirac and Majorana neutrinos if certain mass-specific effects can be observed, i.e., we depend on a parameter whose value is indisputably small. 


\section{Appendix A.4. A Premature Dismissal of Majorana's Ideas}

This Appendix reconstructs the story of a misunderstanding that began in 1957, the effects of which marred the discussion of Majorana's neutrinos for a long time. Let us begin by resuming the present situation. Today, we are convinced that charged weak interactions have a chiral nature, and guided by the principles of the standard model of elementary particles, we think that the only neutrinos needed to discuss these interactions are the left-handed ones. These positions have no implications for the magnitude of the neutrino's Majorana mass, which can be constructed from left-handed neutrinos and which we intend to explore experimentally. To be sure, Equation (12) consistently describes a non-zero Majorana mass in the context of the (extended) standard model.

In 1957, the discussion was at a much earlier stage, and the problem physicists faced was completely different; they had very little idea what the structure of weak interactions was, and they needed to proceed. In order to do so, they decided to try and explore two hypotheses, i.e., (1) the conservation of the leptonic number and also (2) the absence of mass of the neutrino, which were consistent with the experimental facts known at the time. In this way, a strong and useful theoretical simplification of the structure of neutrino interactions was achieved, which moreover implied that the newly discovered parity violation would apply to neutrinos [143-145]. These results clarified an important limiting case, but of course, they did not help to quantify in any way what the size of the neutrino mass is. This point was made very clear in [146], a paper of the same year.

Unfortunately, the three seminal articles on the chiral structure of neutrino interactions (appearing just previously) included very strong statements, which left a lasting impression that masslessness was an inevitable result and not a hypothesis. For example, in [21], we read: "PARITY VIOLATION TAKE PLACES FOR WEAK-DECAYS IN A SPECIFIED MANNER WHICH MAKES THE NEUTRINO SELF-MASS (LIKE THE PHOTON SELF-MASS) VANISH", and in [23], we read: "A MAJORANA THEORY FOR SUCH A NEUTRINO IS THEREFORE IMPOSSIBLE. THE MASS OF THE NEUTRINO AND THE ANTINEUTRINO IN THIS THEORY IS NECESSARILY ZERO". Even in [22] (which states in the Abstract that masslessness is a hypothesis), we read: "THE MASS OF THE LONGITUDINAL NEUTRINO, ON THE OTHER HAND, VANISHES AUTOMATICALLY", which is formally correct, as "longitudinal neutrino" means just a neutrino with fixed helicity in modern parlance, but presents what is a definition as a fact.

Moreover, we must remember the statement against Majorana's mass made in [142] (which, as discussed in Equation (A8), we now consider to be simply wrong), which casts doubt on the most conventional formalism, that of bispinors.

As a subsequent examples of prejudice against Majorana, we quote from the Abstract of an authoritative work of 1959 [147]: “A VERDICT MAY BE TENTATIVELY REACHED IN FAVOR OF A 'DIRAC' NEUTRINO, OPERATIONALLY DISTINGUISHABLE FROM A 'DIRAC' ANTI-NEUTRINO, AND WITH CONSERVATION OF TOTAL LEPTON CHARGE VALID IN ALL NEUTRINO INTERACTIONS" and the words of a beautiful paper appearing in 1963 [148] “KONOPINSKY 1949 ESTIMATED THE TRANSITION PROBABILITIES FOR THE CASES A AND B. (CASE A CORRESPONDS TO DIRAC NEUTRINOS, CASE B TO MAJORANA NEUTRINOS, BOTH WITH FERMI'S INTERACTIONS.) HE CONCLUDED THAT THE PROBABILITY IN CASE B IS ABOUT THOUSAND TIMES LARGER THAN THAT OF CASE A. [...] THIS CONTRADICTS THE EXPERIMENTAL DATA OF DOUBLE BETA DECAY, WHICH FAVOR THE DIRAC THEORY".

The problem was solved only slowly, and we refer to the papers already cited and that appeared in a variety of contexts [27-37]. However, quite surprisingly, the solution was not appreciated universally, and some amount of prejudice continued to linger even in recent times. For example, a justly famous book on nuclear physics [149] appearing in 1998 writes about this: “ONE OF THE INTERESTS IN DOUBLE $\beta$-DECAY [ $\ldots$ ] IS TO FIND OUT WHETHER NEUTRINOS CAN BE MAJORANA PARTICLES. SO FAR ALL THE EVIDENCE SEEMS TO SUGGEST THAT THEY ARE STRICTLY DiRAC PARTICLES", a statement we do not subscribe to for the reasons explained above.

Interestingly, Majorana's mass always continued to be discussed in the literature, but mostly without bispinors, namely adopting Weyl's two-dimensional spinor formalism, as first done in [150] (who apparently was not aware of Majorana), and above all Case's work [27] (who instead recognized Majorana). Case's paper, which again appeared in 1957, had many 
merits, among which was that of ensuring the consideration/survival of Majorana's ideas in the scientific literature also in the new context: this is why we mention it in Table 2. For instance, this work is cited in the classic book [151] and also in the first calculation of nuclear matrix elements for electron creation/double-beta transition of Equation (16) in the context of modern $V-A$ theory [28]. Unfortunately, the use of a formalism unfamiliar to a large part of the scientific community in [27] created the impression that, in order to obtain a coherent field theory, it was necessary to reformulate Majorana's theory with Weyl spinors. However, this is not the case at all: normal bispinors (those with four components) are perfectly fine for this purpose, as argued in the main text and in this Appendix.

A wider and useful discussion of similar issues was given in [113].

\section{References}

1. Feynman, R.P. The reason for anti-particles. In Elementary Particles and the Laws of Physics, The 1986 Dirac Memorial Lectures; Cambridge University Press: Cambridge, UK, 1988.

2. Stueckelberg, E.C.G. La signification du temps propre en mécanique ondulatoire and Remarque à propos de la création de paires de particules en théorie de relativité. Helv. Phys. Acta 1941, 14, 321-322. (In French)

3. Jackson, J.D.; Okun, L.B. Historical roots of gauge invariance. Rev. Mod. Phys. 2001, 73, 663. [CrossRef]

4. Majorana, E. Teoria simmetrica dell'elettrone e del positrone. Nuovo Cim. 1937, 14, 171. (In Italian) [CrossRef]

5. Dirac, P.A.M. The quantum theory of the electron. Proc. R. Soc. Lond. 1928, A117, 610.

6. Dirac, P.A.M. The quantum theory of the electron. Part II. Proc. R. Soc. Lond. 1928, A118, 351.

7. Dirac, P.A.M. Quantised singularities in the electromagnetic field. Proc. R. Soc. Lond. 1931, A133, 60.

8. Gell-Mann, M. A schematic model of baryons and mesons. Phy. Lett. 1964, 8, 214. [CrossRef]

9. Zweig, G. An SU(3) model for strong interaction symmetry and its breaking. CERN-TH-401 \& 412 (1964). In Developments in the Quark Theory of Hadrons; Lichtenberg, D.B., Rosen, S.P., Eds.; Hadronic Press: Palm Harbor, FL, USA, 1980; Volume 1, pp. 1964-1978.

10. Yang, C. Fermi's beta-decay theory. Int. J. Mod. Phys. A 2012, 27, 1230005. [CrossRef]

11. Wigner, E.P. Invariance in physical theory. Proc. Am. Philos. Soc. 1949, 93, 521. [PubMed]

12. Marx, G. Die wechselwirkung der elementar-teilchen und die erhaltungssätze. Acta Phys. Hung. 1953, 3, 55. (In German) [CrossRef]

13. Zel'dovich, Y.B. On the theory of elementary particles. Conservation of the nuclear charge and a possible new type of V-particles. Dokl. Akad. Nauk SSSR 86, 505 (1952). In Selected Works of Ya. B. Zel'dovich, Vol. II: Particles, Nuclei, and the Universe; Princeton University Press: Princeton, NJ, USA, 1993.

14. Konopinski, E.J.; Mahmoud, H.M. The universal Fermi interaction. Phys. Rev. 1953, 92, 1045. [CrossRef]

15. Lee, T. History of the weak interactions. CERN Cour. 1987, 27, 7-12.

16. Weyl, H. Elektron und gravitation. I. Zeitschrift für Physik 1929, 56, 330. (In German) [CrossRef]

17. Stueckelberg, E.C.G. Über die methode der physikalischen naturberschreibung. Naturforschende Gesellschaf Basel Verhandlungen 1936, 47, 181. (In German)

18. Racah, G. Sulla simmetria tra particelle e antiparticelle. Nuovo Cim. 1937, 14, 322. (In Italian) [CrossRef]

19. Lee, T.; Yang, C. Question of parity conservation in weak interactions. Phys. Rev. 1956, 104, 254. [CrossRef]

20. Wu, C.; Ambler, E.; Hayward, R.W.; Hoppes, D.D.; Hudson, R.P. Experimental test of parity conservation in $\beta$ decay. Phys. Rev. 1957, 105, 1413. [CrossRef]

21. Salam, A. On parity conservation and neutrino mass. Nuovo Cim. 1957, 5, 299. [CrossRef]

22. Landau, L.D. On the conservation laws for weak interactions. Nucl. Phys. 1957, 3, 127. [CrossRef]

23. Lee, T.; Yang, C. Parity nonconservation and a two component yheory of the neutrino. Phys. Rev. 1957, 105, 1671. [CrossRef]

24. Goldhaber, M.; Grodzins, L.; Sunyar, A.W. Helicity of neutrinos. Phys. Rev. 1958, 109, 1015. [CrossRef]

25. Sudarshan, E.C.G.; Marshak, R.E. The nature of the four-fermion interaction. Chirality invariance and the universal Fermi interaction. Phys. Rev. 1957, 109, 1860. [CrossRef]

26. Feynman, R.P.; Gell-Mann, M. Theory of Fermi interaction. Phys. Rev. 1958, 109, 193. [CrossRef]

27. Case, K.M. Reformulation of the Majorana theory of the neutrino. Phys. Rev. 1957, 107, 307. [CrossRef]

28. Greuling, E.; Whitten, R.C. Lepton conservation and double-beta decay. Ann. Phys. 1960, 11, 510. [CrossRef]

29. Shchepkin, M.G. On leptonic charge conservation. Yadernaya fizika 1973, 17, 820. (In Russian)

30. Pontecorvo, B.M. Mesonium and anti-mesonium. Sov. Phys. JETP 1957, 6, 429.

31. Bilenky, S.M.; Hošek, J.; Petcov, S.T. On oscillations of neutrinos with Dirac and Majorana masses. Phys. Lett. 1980, B94, 495. [CrossRef]

32. Barbieri, R.; Ellis, J.R.; Galliard, M.K. Neutrino masses and oscillations in SU(5). Phys. Lett. 1980, B90, 249. [CrossRef]

33. Doi, M.; Kotani, T.; Nishiura, H.; Okuda, K.; Takasugi, E. Neutrino masses and the double beta decay. Phys. Lett. 1981, B103, 219. [CrossRef]

34. Li, Li.; Wilczek, F. Physical processes involving Majorana neutrinos. Phys. Rev. 1982, D25, 143. [CrossRef] 
35. Schechter, J.; Valle, J.W.F. Neutrinoless double beta decay in SU(2) $\times \mathrm{U}(1)$ theories. Phys. Rev. 1982, D25, 2951.

36. Kayser, B.; Shrock, R.E. Distinguishing between Dirac and Majorana neutrinos in neutral current reactions. Phys. Lett. 1982, B112, 137. [CrossRef]

37. Kayser, B. Majorana neutrinos and their electromagnetic properties. Phys. Rev. 1982, D26, 1662. [CrossRef]

38. Dell'Oro, S.; Marcocci, S.; Viel, M.; Vissani, F. Neutrinoless double beta decay: 2015 review. Adv. High Energy Phys. 2016, 2016, 2162659. [CrossRef]

39. Pontecorvo, B.M. Pages in the development of neutrino physics. Sov. Phys. Uspekhi 1983, 26, 1087. [CrossRef]

40. Bilenky, S.M.; Blokhintseva, T.D.; Cifarelli, L.; Matveev, V.A.; Pokrovskaya, I.G.; Sapozhnikov, M.G. (Eds.) Bruno Pontecorvo Selected Scientific Works, 2nd ed.; SIF: Bologna, Italy, 2013.

41. Fantini, G.; Rosso, A.G.; Vissani, F.; Zema, V. Introduction to the formalism of neutrino oscillations. Adv. Ser. Direct. High Energy Phys. 2018, 28, 37.

42. Abe, K.; Adam, J.; Aihara, H.; Akiri, T.; Andreopoulos, C.; Aoki, S.; Ariga, A.; Ariga, T.; Assylbekov, S.; Autiero, D.; et al. Observation of electron neutrino appearance in a muon neutrino beam. Phys. Rev. Lett. 2014, 112, 061802. [CrossRef]

43. Adamson, P.; Ader, C.; Andrews, M.; Anfimov, N.; Anghel, I.; Arms, K.; Arrieta-Diaz, E.; Aurisano, A.; Ayres, D.S.; Backhouse, C.; et al. First measurement of electron neutrino appearance in NOvA. Phys. Rev. Lett. 2016, 116, 151806. [CrossRef]

44. Agafonova, N.; Aleksandrov, A.; Anokhina, A.; Aoki, S.; Ariga, A.; Ariga, T.; Asada, T.; Autiero, D.; Dhahbi, A.B.; Badertscher, A.; et al. Evidence for $v_{\mu} \rightarrow v_{\tau}$ appearance in the CNGS neutrino beam with the OPERA experiment. Phys. Rev. 2014, D89, 051102.

45. Abe, K.; Hayato, Y.; Iida, T.; Iyogi, K.; Kameda, J.; Koshio, Y.; Kozuma, Y.; Marti, L.; Miura, M.; Moriyama, S.; et al. Evidence for the appearance of atmospheric tau neutrinos in Super-Kamiokande. Phys. Rev. Lett. 2013, 110, 181802. [CrossRef]

46. Aartsen, M.G.; Ackermann, M.; Adams, J.; Aguilar, J.A.; Ahlers, M.; Ahrens, M.; Altmann, D.; Andeen, K.; Anderson, T.; Ansseau, I.; et al. Measurement of atmospheric tau neutrino appearance with IceCube DeepCore. Phys. Rev. 2017, D99, 032007. [CrossRef]

47. Pontecorvo, B.M. Inverse beta processes and nonconser-vation of lepton charge. Sov. Phys. JETP 1958, 7, 172.

48. Gell-Mann, M.; Pais, A. Behavior of neutral particles under charge conjugation. Phys. Rev. 1955, 97, 1387. [CrossRef]

49. Pontecorvo, B. Neutrino experiments and the problem of conservation of leptonic charge. Sov. Phys. JETP 1968, 26, 984.

50. Katayama, Y.; Matumoto, K.; Tanaka, S.; Yamada, E. Possible unified models of elementary earticles with two neutrinos. Prog. Theor. Phys. 1962, 28, 675. [CrossRef]

51. Maki, Z.; Nakagawa, M.; Sakata, S. Remarks on the unified model of elementary particles. Prog. Theor. Phys. 1962, 28, 870. [CrossRef]

52. Nakagawa, M.; Okonogi, H.; Sakata, S.; Toyoda, A. Possible existence of a neutrino with mass and partial conservation of muon charge. Prog. Theor. Phys. 1963, 30, 727. [CrossRef]

53. Wolfenstein, L. Neutrino oscillations in matter. Phys. Rev. 1978, D17, 2369. [CrossRef]

54. Mikheyev, S.P.; Smirnov, A.Y. Resonance amplification of oscillations in matter and spectroscopy of solar neutrinos. Sov. J. Nucl. Phys. 1985, 42, 913.

55. Cleveland, B.T.; Daily, T.; Davis, R., Jr.; Distel, J.R.; Lande, K.; Lee, C.K.; Wildenhain, P.S.; Ullman. J. Measurement of the solar electron neutrino flux with the Homestake chlorine detector. Astrophys. J. 1998, 496, 505. [CrossRef]

56. Hirata, K.S.; Inoue, K.; Ishida, T.; Kajita, T.; Kihara, K.; Nakahata, M.; Nakamura, K.; Ohara, S.; Sato, N.; Suzuki, Y.; et al. Real time, directional measurement of B-8 solar neutrinos in the Kamiokande-II detector. Phys. Rev. D 1991, 44, 2241. [CrossRef]

57. Kaether, F.; Hampel, W.; Heusser, G.; Kiko, J.; Kirsten, T. Reanalysis of the GALLEX solar neutrino flux and source experiments. Phys. Lett. B 2010, 685, 47. [CrossRef]

58. Abdurashitov, J.N.; Gavrin, V.N.; Gorbachev, V.V.; Gurkina, P.P.; Ibragimova, T.V.; Kalikhov, A.V.; Khairnasov, N.G.; Knodel, T.V.; Mirmov, I.N.; Shikhin, A.A.; et al. Measurement of the solar neutrino capture rate with gallium metal. III: Results for the 2002-2007 data-taking period. Phys. Rev. C 2009, 80, 015807. [CrossRef]

59. Bahcall, J.N. Neutrino Astrophysics; Cambridge University Press: Cambridge, UK, 1989.

60. Bellerive, A.; Klein, J.R.; McDonald, A.B.; Noble, A.J.; Poon, A.W. The Sudbury Neutrino Observatory. Nucl. Phys. B 2016, $908,30$. [CrossRef]

61. Abe, K.; Haga, Y.; Hayato, Y.; Ikeda, M.; Iyogi, K.; Kameda, J.; Kishimoto, Y.; Marti, L.; Miura, M.; Moriyama, S.; et al. Solar neutrino measurements in Super-Kamiokande-IV. Phys. Rev. D 2016, 94, 052010. [CrossRef]

62. Agostini, M.; Altenmuller, K.; Appel, S.; Atroshchenko, V.; Bagdasarian, Z.; Basilico, D. First simultaneous precision spectroscopy of $p p,{ }^{7} \mathrm{Be}$, and pep solar neutrinos with Borexino phase-II. Phys. Rev. D 2019, 100, 082004. [CrossRef]

63. Gando, A.; Gando, Y.; Hanakago, H.; Ikeda, H.; Inoue, K.; Ishidoshiro, K.; Ishikawa, H.; Koga, M.; Matsuda, R.; Matsuda, S.; et al. Reactor on-off antineutrino measurement with KamLAND. Phys. Rev. D 2013, 88, 033001. [CrossRef]

64. Hirata, K.S. Observation of a small atmospheric muon-neutrino/electron-neutrino ratio in Kamiokande. Phys. Lett. B 1992, 280, 146. [CrossRef]

65. Fukuda, Y. Atmospheric muon-neutrino/electron-neutrino ratio in the multiGeV energy range. Phys. Lett. B 1994, 335, 237. [CrossRef]

66. Fukuda, Y.; Hayakawa, T.; Ichihara, E.; Inoue, K.; Ishihara, K.; Ishino, H.; Itow, Y.; Kajita, T.; Kameda, J.; Kasuga, S.; et al. Evidence for oscillation of atmospheric neutrinos. Phys. Rev. Lett. 1998, 81, 1562. [CrossRef] 
67. Ambrosio, M.; Antolini, R.; Aramo, C.; Auriemma, G.; Baldini, A.; Barbarino, G.C.; Barish, B.C.; Battistoni, G.; Bellotti, R.; Bemporad, C.; et al. Measurement of the atmospheric neutrino induced upgoing muon flux using MACRO. Phys. Lett. B 1998, 434, 451. [CrossRef]

68. Allison, W.W.; Alner, G.J.; Ayres, D.S.; Barr, G.; Barrett, W.L.; Bode, C.; Border, P.M.; Brooks, C.B.; Cobb, J.H.; Cotton, R.J.; et al. The atmospheric neutrino flavor ratio from a 3.9 fiducial kiloton year exposure of Soudan-2. Phys. Lett. B 1999, 449, 137. [CrossRef]

69. Ahn, M.H.; Aoki, S.; Bhang, H.; Boyd, S.; Casper, D.; Choi, J.H.; Fukuda, S.; Fukuda, Y.; Gajewski, W.; Hara, T.; et al. Indications of neutrino oscillation in a $250 \mathrm{~km}$ long baseline experiment. Phys. Rev. Lett. 2003, 90, 041801. [CrossRef]

70. Adey, D.; An F.P.; Balantekin, A.B.; Balantekin, H.R.; Bishai, M.; Blyth, S.; Cao, D.; Cao, G.F.; Cao, J.; Chan, Y.L.; et al. Measurement of electron antineutrino oscillation with 1958 days of operation at Daya Bay. Phys. Rev. Lett. 2018, 121, 241805. [CrossRef] [PubMed]

71. Bak, G.; Choi, J.H.; Jang, H.I.; Jang, J.S.; Jeon, S.H.; Joo, K.K.; Ju, K.; Jung, D.E.; Kim, J.G.; Kim, J.H.; et al. Measurement of reactor antineutrino oscillation amplitude and frequency at RENO. Phys. Rev. Lett. 2018, 121, 201801. [CrossRef]

72. De Kerret, H. First Double Chooz $\theta_{13}$ measurement via total neutron capture detection. Nat. Phys. 2020, $16,558$.

73. Capozzi, F.; Lisi, E.; Marrone, A.; Palazzo, A. Current unknowns in the three neutrino framework. Prog. Part Nucl. Phys. 2018, 102, 48. [CrossRef]

74. De Salas, P.F.; David; Forero, V.; Ternes, C.A.; Tórtola, M.; Valle, J.W.F. Phys. Lett. 2018, B782, 633. [CrossRef]

75. Esteban, I.; González-García, M.C.; Hernández-Cabezudo, A.; Maltoni, M.; Schwetz, T. Global analysis of three-flavour neutrino oscillations: Synergies and tensions in the determination of $\theta_{23}, \delta_{C P}$, and the mass ordering. J. High Energy Phys. 2019, 106. [CrossRef]

76. Esteban, I.; González-García, M.C.; Maltoni, M.; Schwetz, T.; Zhou, A. The fate of hints: Updated global analysis of three-flavor neutrino oscillations. J. High Energy Phys. 2020, 178. [CrossRef]

77. Abe, K.; Aihara, H.; Andreopoulos, C.; Anghel, I.; Ariga, A.; Ariga, T.; Asfandiyarov, R.; Askins, M.; Back, J.J.; Ballett, P.; et al. Physics potential of a long-baseline neutrino oscillation experiment using a J-PARC neutrino beam and Hyper-Kamiokande. Prog. Theor. Exp. Phys. 2015, 2015, 053C02.

78. An F.; An G.; An Q.; Antonelli, V.; Baussan, E.; Beacom, J.; Bezrukov, L.; Blyth, S.; Brugnera, R.; Avanzini, M.B.; et al. Neutrino physics with JUNO. J. Phys. G 2016, 43, 030401. [CrossRef]

79. Abi, B. Deep underground neutrino experiment (DUNE), far detector technical design report, Vol. II DUNE physics. arXiv 2020, arXiv:2002.03005.

80. Glashow, S.L. Partial symmetries of weak interactions. Nucl. Phys. 1961, 22, 579. [CrossRef]

81. Weinberg, S. A model of leptons. Phys. Rev. Lett. 1967, 19, 1264. [CrossRef]

82. Salam, A. Weak and electromagnetic interactions. In Proceedings of the 8th Nobel Symposium, Aspenasgarden, Lerum, 19-25 May 1968; Volume 367.

83. Bardeen, W. Anomalous Ward identities in spinor field theories. Phys. Rev. 1969, 184, 1848. [CrossRef]

84. Bouchiat, C.; Iliopoulos, J.; Meyer, P. An anomaly-free version of Weinberg's model. Phys. Lett. B 1972, 38, 519. [CrossRef]

85. Hooft, G.; Veltman, M.J.G. Regularization and renormalization of gauge fields. Nucl. Phys. B 1972, 44, 189. [CrossRef]

86. Hooft, G. Symmetry breaking through Bell-Jackiw anomalies. Phys. Rev. Lett. 1976, 37, 8. [CrossRef]

87. Kuzmin, V.A.; Rubakov, V.A.; Shaposhnikov, M.E. On anomalous electroweak baryon-number non-conservation in the early universe. Phys. Lett. 1985, B155, 36. [CrossRef]

88. Kajantie, K.; Laine, M.; Rummukainen, K.; Shaposhnikov, M.E. Is there a hot electroweak phase transition at $m_{H}$ larger or equal to $m_{W}$ ? Phys. Rev. Lett. 1996, 77, 2887. [CrossRef]

89. Minkowski, P. $\mu \rightarrow e \gamma$ at a rate of 1 out of $10^{9}$ muon decays? Phys. Lett. 1977, B67, 421. [CrossRef]

90. Yanagida, T. Horizontal gauge symmetry and masses of neutrinos. In Proceedings of the Workshop on the Baryon Number of the Universe and Unified Theories, Tsukuba, Japan, 13-14 February 1979; Volume 95.

91. Glashow, S.L. Overview. Summary Talk at Neutrino 1979, Bergen, Norway, 18-22 June 1979.

92. Gell-Mann, M.; Ramond, P.; Slansky, R. Complex spinors and unified theories. In Proceedings of the Supergravity Workshop, Stony Brook, NY, USA, 27-29 September 1979; Volume 315.

93. Mohapatra, R.N.; Senjanovic, G. Neutrino mass and spontaneous parity nonconservation. Phys. Rev. Lett. 1980, 44, 912. [CrossRef]

94. Pati, J.C.; Salam, A. Unified lepton-hadron symmetry and a gauge theory of the basic interactions. Phys. Rev. D 1973, 8,1240 . [CrossRef]

95. Pati, J.C.; Salam, A. Lepton number as the fourth color. Phys. Rev. D 1974, 10, 275. [CrossRef]

96. Fukugita, M.; Yanagida, T. Baryogenesis without grand unification. Phys. Lett. 1986, B174, 45. [CrossRef]

97. Weinberg, S. Baryon and lepton nonconserving processes. Phys. Rev. Lett. 1979, 43, 1566. [CrossRef]

98. Wilczek, F.; Zee, A. Operator analysis of nucleon decay. Phys. Rev. Lett. 1979, 43, 1571. [CrossRef]

99. Touschek, B. Zur theorie des doppelten $\beta$-zerfalls. Zeitschrift für Physik 1948, 125, 108. (In German) [CrossRef]

100. Inghram, M.G.; Reynolds, J.H. Double beta-decay of Te-130. Phys. Rev. 1950, 78, 822. [CrossRef]

101. Fiorini, E.; Pullia, A.; Bertolini, G.; Cappellani, F.; Restelli, G. A search for lepton nonconservation in double $\beta$ decay with a germanium detector. Phys. Lett. 1967, B25, 602. [CrossRef]

102. Bellotti, E.; Cremonesi, O.; Fiorini, E.; Liguori, C.; Ragazzi, S. Multielement proportional chamber for Xe-136 $\beta$ decay. AIP Conf. Proc. 1984, 108, 42. 
103. Miyajima, M.; Sasaki, S.; Tawara, H. Search for double beta decay products of Xe-136 in liquid xenon. IEEE Trans. Nucl. Sci. 1994, 41, 835. [CrossRef]

104. Tretyak, V.I.; Zdesenko, Y.G. Tables of double beta decay data: An update. At. Data Nucl. Data Tables 2002, 80, 83. [CrossRef]

105. Zdesenko, Y.G. The future of double beta decay research. Rev. Mod. Phys. 2003, 74, 663. [CrossRef]

106. Vergados, J.D.; Ejiri, H.; Šimkovic, F. Neutrinoless double beta decay and neutrino mass. Int. J. Mod. Phys. E 2016, 25, 1630007. [CrossRef]

107. Engel, J.; Menéndez, J. Status and future of nuclear matrix elements for neutrinoless double-Beta decay: A review. Rep. Prog. Phys. 2017, 80, 046301. [CrossRef] [PubMed]

108. Dolinski, M.J.; Poon, A.W.P.; Rodejohann, W. Neutrinoless double-beta decay: Status and prospects. Ann. Rev. Nucl. Part. Sci. 2019, 69, 219. [CrossRef]

109. Tretyak, V.I. False starts in history of searches for $2 \beta$ decay, or discoverless double beta decay. AIP Conf. Proc. 2011, 1417, 129.

110. Davis, R., Jr. Attempt to detect the antineutrinos from a nuclear reactor by the ${ }^{37} \mathrm{Cl}\left(\bar{v}, e^{-}\right){ }^{37}$ Ar reaction. Phys. Rev. 1955, 97,766 . [CrossRef]

111. Furry, W.H. On transition probabilities in double beta-disintegration. Phys. Rev. 1939, 56, 1184. [CrossRef]

112. Goeppert-Mayer, M. Double beta-disintegration. Phys. Rev. 1935, 48, 512. [CrossRef]

113. Bianchi, S.D. Rethinking antiparticles. Hermann Weyl's contribution to neutrino physics. Stud. Hist. Philos. Mod. Phys. 2018, 61, 68. [CrossRef]

114. Klapdor-Kleingrothaus, H.V. Sixty Years of Double Beta Decay: From Nuclear Physics to Beyond Standard Model Particle Physics; World Scientific: Singapore, 2001.

115. Fogli, G.L.; Lisi, E.; Montanino, D. A comprehensive analysis of solar, atmospheric, accelerator and reactor neutrino experiments in a hierarchical three generation scheme. Phys. Rev. 1994, D49, 3626. [CrossRef] [PubMed]

116. Capozzi, F.; Valentino, E.D.; Lisi, E.; Marrone, A.; Melchiorri, A.; Palazzo, A. Global constraints on absolute neutrino masses and their ordering. Phys. Rev. 2017, D95, 096014. [CrossRef]

117. Gando, A.; Gando, Y.; Hachiya, T.; Hayashi, A.; Hayashida, S.; Ikeda, H.; Inoue, K.; Ishidoshiro, K.; Karino, Y.; Koga, M.; et al. Search for Majorana neutrinos near the inverted mass hierarchy region with KamLAND-Zen. Phys. Rev. Lett. 2016, 117, 082503. [CrossRef] [PubMed]

118. Alduino, C.; Alessandria, F.; Alfonso, K.; Andreotti, E.; Arnaboldi, C.; Avignone, F.T., III; Azzolini, O.; Balata, M.; Bandac, I.; Banks, T.I.; et al. First results from CUORE: A search for lepton number violation via $0 v \beta \beta$ decay of ${ }^{130} \mathrm{Te}$. Phys. Rev. Lett. 2018, 120, 132501. [CrossRef] [PubMed]

119. Azzolini, O.; Beeman, J.W.; Bellini, F.; Beretta, M.; Biassoni, M.; Brofferio, C.; Bucci, C.; Capelli, S.; Cardani, L.; Carniti, P.; et al. Final result of CUPID-0 phase-I in the search for the ${ }^{82}$ Se neutrinoless double- $\beta$ decay. Phys. Rev. Lett. 2019, $123,032501$. [CrossRef] [PubMed]

120. Agostini, M.; Araujo, G.R.; Bakalyarov, A.M.; Balata, M.; Barabanov, I.; Baudis, L.; Bauer, C.; Bellotti, E.; Belogurov, S.; Bettini, A.; et al. Final results of GERDA on the search for neutrinoless double- $\beta$ decay. Phys. Rev. Lett. 2020, $125,252502$. [CrossRef] [PubMed]

121. Vissani, F. Signal of neutrinoless double beta decay, neutrino spectrum and oscillation scenarios. J. High Energy Phys. 1999, 1999, 022. [CrossRef]

122. Shrock, R.E. New tests for, and bounds on, neutrino masses and lepton mixing. Phys. Lett. 1980, B96, 159. [CrossRef]

123. McKellar, B.H.J. The Influence of mixing of finite mass neutrinos on beta decay spectra. Phys. Lett. 1980, B97, 93. [CrossRef]

124. Kobzarev, I.Y.; Martemyanov, B.V.; Okun, L.B.; Shchepkin, M.G. The phenomenology of neutrino oscillations. Sov. J. Nucl. Phys. 1980, 32, 823.

125. Vissani, F. Nonoscillation searches of neutrino mass in the age of oscillations. Nucl. Phys. B Proc. Suppl. 2001, 100, 273. [CrossRef]

126. Farzan, Y.; Smirnov, A.Y. On the effective mass of the electron neutrino in beta decay. Phys. Lett. 2003, B557, 224. [CrossRef]

127. Aker, M.; Altenmüller, K.; Arenz, M.; Babutzka, M.; Barrett, J.; Bauer, S.; Beck, M.; Beglarian, A.; Behrens, J.; Bergmann, T.; et al. Improved upper limit on the neutrino mass from a direct kinematic method by KATRIN. Phys. Rev. Lett. 2019, 123, 221802. [CrossRef] [PubMed]

128. Fogli, G.; Lisi, E.; Marrone, A.; Melchiorri, A.; Palazzo, A.; Serra, P.; Silk, J. Observables sensitive to absolute neutrino masses: Constraints and correlations from world neutrino data. Phys. Rev. 2004, D70, 113003. [CrossRef]

129. Aghanim, N.; Akrami, Y.; Ashdown, M.; Aumont, J.; Baccigalupi, C.; Ballardini, M.; Banday, A.J.; Barreiro, R.B.; Bartolo, N.; Basak, S.; et al. Planck 2018 results. VI. Cosmological parameters. Astron. Astrophys. 2020, 641, A6.

130. Yèche, C.; Palanque-Delabrouille, N.; Baur, J.; Des Bourboux, H.D.M. Constraints on neutrino masses from Lyman-alpha forest power spectrum with BOSS and XQ-100. J. Cosmol. Astropart. Phys. 2017, 6, 047. [CrossRef]

131. Palanque-Delabrouille, N.; Yèche, C.; Schöneberg, N.; Lesgourgues, J.; Walther, M.; Chabanier, S. Hints, neutrino bounds and WDM constraints from SDSS DR14 Lyman- $\alpha$ and Planck full-survey data. J. Cosmol. Astropart. Phys. 2020, 4, 038. [CrossRef]

132. Agostini, M.; Benato, G.; Dell’Oro, S.; Pirro, S.; Vissani, F. Discovery probabilities of Majorana neutrinos based on cosmological data. Phys. Rev. 2021, D103, 033008.

133. Dell'Oro, S.; Marcocci, S.; Vissani, F. Empirical Inference on the Majorana Mass of the Ordinary Neutrinos. Phys. Rev. 2019, D100, 073003. [CrossRef] 
134. Dell'Oro, S.; Marcocci, S.; Viel, M.; Vissani, F. The contribution of light Majorana neutrinos to neutrinoless double $\beta$ decay and cosmology. J. Cosmol. Astropart. Phys. 2015, 12, 023. [CrossRef]

135. Capozzi, F.; Valentino, E.D.; Lisi, E.; Marrone, A.; Melchiorri, A.; Palazzo, A. Addendum to “Global constraints on absolute neutrino masses and their ordering". Phys. Rev. 2020, D101, 116013.

136. Serpe, J.N.F.J. Sur la théorie abrégée des particules de spin 1/2. Physica 1952, 18, 295. (In French) [CrossRef]

137. Feinberg, G.; Goldhaber, M. Microscopic tests of symmetry principles. Proc. Natl. Acad. Sci. USA 1959, 45, 1301. [CrossRef]

138. Pontecorvo, B. Superweak interactions and double beta decay. Phys. Lett. 1968, B26, 630. [CrossRef]

139. Vissani, F. Majorana Neutrinos: What, Why, Where? Invit. Sem. at the WIN Meeting, Bari, 2019. Available online: https:// agenda.infn.it/event/13938/contributions/91583/attachments/64054/77749/vissani-win-2019.pdf (accessed on 2 March 2021).

140. Vissani, F. A History of Some Recent Attempts to Go Beyond the Standard model. Invit. Sem. at the Workshop Gravity, Information and Fundamental Symmetries of the Max Planck Society, Munich, 2019. Available online: https://www.mpi-hd. mpg.de/lin/events/ra-workshop/talks/Vissani-sm.pdf (accessed on 2 March 2021).

141. Vissani, F. Cos'è la Materia Per la Fisica Delle Particelle e Perché Tentare di Osservarne la Creazione. Invit. Sem. at the Meeting of the Italian Physics Society (SIF), Milan, 2020. Available online: https://www.sif.it/static/SIF/resources/public/files/congr20/ ri/Vissani.pdf (accessed on 2 March 2021). (In Italian)

142. McLennan, J.A., Jr. Parity nonconservation and the theory of the neutrino. Phys. Rev. 1957, 106, 821. [CrossRef]

143. Pursey, D.L. Invariance properties of Fermi interactions. Nuovo Cim. 1957, 6, 204. [CrossRef]

144. Pauli, W. On the conservation of the lepton charge. Nuovo Cim. 1957, 6, 250. [CrossRef]

145. Enz, C.D. Fermi interaction with non-conservation of «lepton charge» and of parity. Nuovo Cim. 1957, 6, 266. [CrossRef]

146. Radicati, L.A.; Touschek, B. On the equivalence theorem for the massless neutrino. Nuovo Cim. 1957, 6, 1694. [CrossRef]

147. Primakoff, H.; Rosen, S.P. Double beta decay. Rep. Prog. Phys. 1959, 22, 121. [CrossRef]

148. Morita, M. Theory of beta decay. Prog. Theor. Phys. Suppl. 1963, 26, 1. [CrossRef]

149. Wong, S.S.M. Introductory Nuclear Physics; Wiley: New York, NY, USA, 1998.

150. Jehle, H. Two-component wave equations. Phys. Rev. 1949, 75, 1609. [CrossRef]

151. Fukugita, M.; Yanagida, T. Physics of Neutrinos and Applications to Astrophysics; Springer: Berlin, Germany, 2003. 